\title{
Semiconservative replication in the quasispecies model
}

\section{Citation}

Tannenbaum, Emmanuel, Eric J. Deeds, and Eugene I. Shakhnovich. 2004. “Semiconservative Replication in the Quasispecies Model." Physical Review E69 (6): 061916. https://doi.org/10.1103/ PhysRevE.69.061916.

\section{Permanent link}

http://nrs.harvard.edu/urn-3:HUL.InstRepos:41534494

\section{Terms of Use}

This article was downloaded from Harvard University's DASH repository, and is made available under the terms and conditions applicable to Other Posted Material, as set forth at http:// nrs.harvard.edu/urn-3:HUL.InstRepos:dash.current.terms-of-use\#LAA

\section{Share Your Story}

The Harvard community has made this article openly available.

Please share how this access benefits you. Submit a story.

Accessibility 


\title{
Semiconservative replication in the quasispecies model
}

\author{
Emmanuel Tannenbaum, ${ }^{*}$ Eric J. Deeds, and Eugene I. Shakhnovich \\ Harvard University, Cambridge, Massachusetts 02138, USA \\ (Received 28 September 2003; revised manuscript received 23 January 2004; published 16 June 2004)
}

\begin{abstract}
This paper extends Eigen's quasispecies equations to account for the semiconservative nature of DNA replication. We solve the equations in the limit of infinite sequence length for the simplest case of a static, sharply peaked fitness landscape. We show that the error catastrophe occurs when $\mu$, the product of sequence length and per base pair mismatch probability, exceeds $2 \ln [2 /(1+1 / k)]$, where $k>1$ is the first-order growth rate constant of the viable "master" sequence (with all other sequences having a first-order growth rate constant of 1). This is in contrast to the result of $\ln k$ for conservative replication. In particular, as $k \rightarrow \infty$, the error catastrophe is never reached for conservative replication, while for semiconservative replication the critical $\mu$ approaches $2 \ln 2$. Semiconservative replication is therefore considerably less robust than conservative replication to the effect of replication errors. We also show that the mean equilibrium fitness of a semiconservatively replicating system is given by $k\left(2 e^{-\mu / 2}-1\right)$ below the error catastrophe, in contrast to the standard result of $k e^{-\mu}$ for conservative replication (derived by Kimura and Maruyama in 1966). From this result it is readily shown that semiconservative replication is necessary to account for the observation that, at sufficiently high mutagen concentrations, faster replicating cells will die more quickly than more slowly replicating cells. Thus, in contrast to Eigen's original model, the semiconservative quasispecies equations are able to provide a mathematical basis for explaining the efficacy of mutagens as chemotherapeutic agents.
\end{abstract}

DOI: 10.1103/PhysRevE.69.061916

PACS number(s): 87.23.Kg, 87.16.Ac, 64.90.+b

\section{INTRODUCTION}

In 1971, Manfred Eigen introduced the quasispecies formulation of molecular evolution to explain the observed distribution of genotypes in RNA evolution experiments $[1,2]$. The central result of his model was that due to mutations, the equilibrium distribution of genotypes did not consist of a fittest sequence, but rather a set of closely related strains, which Eigen termed a "quasispecies." Eigen showed that a stable quasispecies only exists if the mutation rate is kept below a threshold value. Above this value, the distribution of genotypes undergoes a phase transition termed the error catastrophe, in which the distribution completely delocalizes over the gene sequence space. Subsequent studies on the quasispecies model have focused almost exclusively on the error catastrophe [3-10], though there has also been some work on the dynamical aspects of the equations [11,12]. More recently, other phase transitions besides the error catastrophe (e.g., the so-called "repair catastrophe") have been shown to arise from the quasispecies equations [13,14].

A common feature of previous work on the quasispecies equations has been the implicit assumption that the genome of an organism could be written as a linear symbol sequence, and that replication occurs conservatively (that is, the original genetic material is preserved during replication). These two assumptions allow for a relatively straightforward derivation of a system of equations modeling the evolution of a unicellular, asexual population. In the simplest formulation, we assume that each organism has a genome $\sigma=s_{1} s_{2} \ldots s_{L}$ of length $L$, where each "letter" or "base" $s_{i}$ is drawn from an alphabet of size $S$ (=4 for all known terrestrial life). We

*Electronic address: etannenb@fas.harvard.edu assume first-order growth kinetics, and that the genome determines the first-order growth rate constant, or fitness, denoted by $\kappa_{\sigma}$ (in general, $\kappa_{\sigma}$ will be time dependent, reflecting the generally dynamic nature of the environment). If we let $x_{\sigma}$ denote the fraction of organisms with genome $\sigma$, then it may be shown that $[1,2]$

$$
\frac{d x_{\sigma}}{d t}=\sum_{\sigma^{\prime}} \kappa_{m}\left(\sigma^{\prime}, \sigma\right) x_{\sigma^{\prime}}-\bar{\kappa}(t) x_{\sigma},
$$

where $\bar{\kappa}(t) \equiv \Sigma_{\sigma} \kappa_{\sigma} x_{\sigma}$ is simply the mean fitness of the population and $\kappa_{m}\left(\sigma^{\prime}, \sigma\right)$ is the first-order mutation rate constant for mutations from $\sigma^{\prime}$ to $\sigma$. If $p_{m}\left(\sigma^{\prime}, \sigma\right)$ denotes the probability of mutation from $\sigma^{\prime}$ to $\sigma$, then it is clear that $\kappa_{m}\left(\sigma^{\prime}, \sigma\right)=\kappa_{\sigma^{\prime}} p_{m}\left(\sigma^{\prime}, \sigma\right)$. To compute $p_{m}\left(\sigma^{\prime}, \sigma\right)$, we assume a per base replication error probability of $\epsilon_{\sigma}$. If we let $D_{H}\left(\sigma^{\prime}, \sigma\right)$ denote the Hamming distance between $\sigma^{\prime}$ and $\sigma$, then it is possible to show that

$$
p_{m}\left(\sigma^{\prime}, \sigma\right)=\left(\frac{\epsilon_{\sigma^{\prime}}}{S-1}\right)^{D_{H}\left(\sigma^{\prime}, \sigma\right)}\left(1-\epsilon_{\sigma^{\prime}}\right)^{L-D_{H}\left(\sigma^{\prime}, \sigma\right)} .
$$

The simplest formulation of these equations considers a genome-independent replication error probability $\epsilon$, and a time-independent fitness landscape characterized by a single "master" sequence $\sigma_{0}$ of fitness $k>1$, with all other sequences set to a fitness of 1 . This so-called single fitness peak (SFP) model has been the subject of considerable theoretical treatment [3-5] (and references therein). The central result of this model is that, in the limit of $L \rightarrow \infty$, the mean equilibrium fitness of the population is given by $k e^{-\mu}$ for $\mu$ $\leqslant \ln k$, and 1 for $\mu>\ln k$, where $\mu \equiv L \epsilon$. When $\mu<\ln k$, the population is localized in a cluster about the master sequence, resulting in what Eigen called a quasispecies. When 
$\mu>\ln k$, the population is completely delocalized over the gene sequence space, so that no discernible quasispecies exists. The transition between the two regimes, at $\mu_{\text {crit }} \equiv \ln k$, is known as the error catastrophe. It should be noted that the result of $k e^{-\mu}$ was first derived in 1966 by Kimura and Maruyama [15], and is a standard result in theoretical population genetics.

While the assumption of a linear symbol sequence and conservative replication is correct for modeling singlestranded RNA, a proper extension of the quasispecies model to real organisms should take into account the doublestranded nature of DNA, and also the semiconservative nature of DNA replication. In semiconservative replication, the original DNA molecule is not preserved after replication. Rather, each strand serves as the template for the synthesis of a complementary daughter strand, meaning that after replication, each DNA molecule consists of one parent and one daughter strand [16].

The formulation of the quasispecies equations given above are inadequate to describe evolution with doublestranded, semiconservatively replicated genomes. There are several reasons for this: First of all, because DNA is double stranded, there is no well-defined Hamming distance between two DNA molecules. Secondly, because daughter strand synthesis occurs off of two parent templates, a single DNA genome gives rise to two DNA daughter genomes, while in conservative replication only one new genome is produced per replication cycle. Finally, because in semiconservative replication the original molecule is destroyed, a mathematical formulation of this process must incorporate an effective death term, which is clearly lacking in the quasispecies equations for conservative replication.

The goal of this paper is to extend Eigen's formulation of the quasispecies equations, to account for the doublestranded and semiconservative nature of DNA replication. This is a necessary first step toward making the quasispecies equations a quantitative tool for analyzing the evolutionary dynamics of unicellular organisms. Then, after obtaining the form of Eigen's equations for the case of double-stranded DNA, we wish to proceed and solve these equations for the simplest landscape, that of the static single fitness peak.

This paper is organized as follows. In the following section, we present an overview of DNA sequence analysis and replication mechanism, followed by a derivation of the appropriate quasispecies equations. We continue in Sec. III with a discussion of the single fitness peak model. Specifically, we present the infinite sequence length equations, leaving the details of the derivation, which are fairly involved, for Appendix A. We then go on to discuss the error catastrophe, presenting both analytical results and numerical corroboration using stochastic simulations of replicating populations. In Sec. IV, we discuss our results, and also the extension of our equations to multiple gene models. Finally, we conclude in Sec. V with a summary of our results, and a discussion of future research plans.

\section{DERIVATION OF THE QUASISPECIES EQUATIONS FOR SEMICONSERVATIVE REPLICATION}

\section{A. An overview of DNA sequence analysis}

Double-stranded DNA consists of two antiparallel, complementary strands. During transcription, messenger

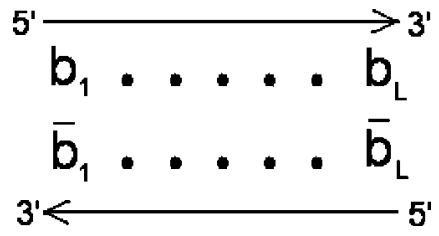

FIG. 1. The antiparallel nature of double-stranded DNA and RNA.

RNA (mRNA) is synthesized in the $5^{\prime}$ to $3^{\prime}$ direction. The DNA template strand from which RNA synthesis occurs is known as the antisense strand, and is read in the $3^{\prime}$ to $5^{\prime}$ direction. The complementary strand, the sense strand, has the same sequence as the transcribed mRNA, and is "read" in the $5^{\prime}$ to $3^{\prime}$ direction (the quotes are to indicate that the sense strand does not directly participate in the transcription process). We therefore adopt the convention that DNA and RNA sequences are read in the $5^{\prime}$ to $3^{\prime}$ direction, as illustrated in Fig. 1. However, this convention is arbitrary, and it is equally valid to read DNA and RNA sequences in the $3^{\prime}$ to $5^{\prime}$ directions. Once a convention is adopted, the antiparallel nature of double-stranded DNA (or RNA) means that the complementary strands are read in opposite directions. A more detailed explanation can be found in Ref. [16].

We consider a double-stranded DNA molecule with generalized alphabet of size $2 S$, consisting of "letters" $0,1, \ldots, 2 S-1$. Each "letter" $i$ is assumed to uniquely pair with $(i+S) \bmod 2 S$. For actual DNA, we of course have $S$ $=2$, and we may make the assignment $\mathrm{A} \rightarrow 0, \mathrm{G} \rightarrow 1, \mathrm{~T} \rightarrow 2$, $\mathrm{C} \rightarrow 3$.

Given a DNA molecule of sequence length $L$, let one of the strands be denoted by $\sigma=b_{1} \cdots b_{L}$. If the complement of a base $b_{i}$ is denoted by $\bar{b}_{i}$, then the complementary strand is given by $\bar{\sigma}=\bar{b}_{L} \cdots \bar{b}_{1}$. Note that $\overline{\bar{\sigma}}=\sigma$, and therefore, each DNA molecule may be denoted by the set $\{\sigma, \bar{\sigma}\}=\{\bar{\sigma}, \sigma\}$.

For single-stranded molecules of length $L$ and alphabet size $2 S$, there are $(2 S)^{L}$ distinct sequences. We seek to derive the analogous formula for double-stranded DNA. Given a DNA molecule $\{\sigma, \bar{\sigma}\}$, define the internal Hamming distance $l_{I}=D_{H}(\sigma, \bar{\sigma})$. If we let $N_{I}\left(l_{I} ; L\right)$ denote the number of DNA molecules of length $L$ with internal Hamming distance $l_{l}$, then the total number of distinct sequences is simply given by $N_{\text {tot }}=\sum_{l_{l}=0}^{L} N_{I}\left(l_{I} ; L\right)$. We therefore proceed to compute $N_{I}\left(l_{I} ; L\right)$. Due to the possibility of palindromic molecules $(\sigma=\bar{\sigma})$, we need to consider the case of $L$ even and $L$ odd separately.

Given some DNA molecule $\{\sigma, \bar{\sigma}\}$, with $\sigma=b_{1} \cdots b_{L}$, suppose we have $b_{i}=\bar{b}_{L-i+1}$ for some $i$. Then $\bar{b}_{i}=b_{L-i+1}$, and hence equality between corresponding bases in $\sigma$ and $\bar{\sigma}$ comes in pairs whenever $i \neq L-i+1$. This must always be true, since, if $i=L-i+1$, then $b_{i}=\bar{b}_{L-i+1} \Rightarrow b_{i}=\bar{b}_{i}$, which is impossible. Therefore, $\sigma$ and $\bar{\sigma}$ must be equal at an even number of sites, hence $l_{I}$ must be odd for odd $L$ and even for even $L$.

Suppose $L$ is odd, so $L=2 l+1$, and consider some $l_{I}=2 k$ +1 . We have complete freedom to choose $b_{1}, \ldots, b_{l+1}$. We automatically have $b_{l+1} \neq \bar{b}_{L-l-1+1}$. Thus, we have $l-k$ re- 
maining sites among $b_{1}, \ldots, b_{l}$ where we choose $b_{L-i+1}$ such that $\bar{b}_{L-i+1}=b_{i}$. Equivalently, we have $k$ sites among $b_{1}, \ldots, b_{l}$ where we choose $b_{L-i+1}$ such that $\bar{b}_{L-i+1} \neq b_{i}$. There are $\left(\begin{array}{l}l \\ k\end{array}\right)$ ways of choosing these sites, and for each such choice, there are $2 S-1$ possible values for each $b_{L-i+1}$ taken to be distinct from $\bar{b}_{i}$. Putting together all the degeneracies, we obtain $\left(\begin{array}{l}l \\ k\end{array}\right)$ $\times(2 S)^{l+1}(2 S-1)^{k}$ ways of choosing $\sigma$ such that $D_{H}(\sigma, \bar{\sigma})$ $=2 k+1=l_{I}$. However, this still does not give us the set of all distinct DNA molecules $\{\sigma, \bar{\sigma}\}$ with internal Hamming distance $l_{I}$, for if $\sigma \neq \bar{\sigma}$, then our counting method generates a given $\{\sigma, \bar{\sigma}\}$ twice, by generating both $\sigma$ and $\bar{\sigma}$. Since $\sigma=\bar{\sigma}$ if and only if $l_{I}=0$, which is impossible for odd $L$, we have, finally, that

$$
N_{I}\left(l_{I}=2 k+1 ; L\right)=\frac{1}{2}\left(\begin{array}{l}
l \\
k
\end{array}\right)(2 S)^{l+1}(2 S-1)^{k}
$$

for odd $L$. Thus, for odd $L$,

$$
N_{\text {tot }}=\frac{1}{2}(2 S)^{l+1} \sum_{k=0}^{l}\left(\begin{array}{l}
l \\
k
\end{array}\right)(2 S-1)^{k}=\frac{1}{2}(2 S)^{L} .
$$

If $L$ is even, then we may write $L=2 l$. In this case, $l_{I}$ is also even, and so $l_{I}=2 k$ for some $k=0, \ldots, l$. We have complete freedom to choose $b_{1}, \ldots, b_{l}$. Proceeding as with the analysis above, we may show that there are $\left(\begin{array}{l}l \\ k\end{array}\right)(2 S)^{l}(2 S-1)^{k}$ ways of choosing $\sigma$ so that $D_{H}(\sigma, \bar{\sigma})=l_{I}$. If $l_{I} \neq 0$, we need to divide by 2 to get the set of all distinct DNA molecules with internal Hamming distance $l_{I}$. Therefore,

$$
N_{I}\left(l_{I}=2 k ; L\right)=\left\{\begin{array}{l}
\frac{1}{2}\left(\begin{array}{l}
l \\
k
\end{array}\right)(2 S)^{l}(2 S-1)^{k} \text { for } k \neq 0, \\
(2 S)^{l} \text { for } k=0
\end{array}\right.
$$

for even $L$. Therefore, for even $L$,

$$
N_{\text {tot }}=\frac{1}{2}(2 S)^{L}+\frac{1}{2}(2 S)^{L / 2} .
$$

Note the additional $1 / 2(2 S)^{L / 2}$ term arising from the contribution of the palindromic sequences.

\section{B. Modeling DNA replication}

The replication of DNA during cell division may be divided into three stages, which are illustrated in Fig. 2. The first stage of DNA replication is strand separation, with each parent strand serving as a template for synthesizing the complementary daughter strands [16]. We may model this stage by writing that a given DNA molecule $\{\sigma, \bar{\sigma}\}$ separates into the single-stranded sequences $\sigma$ and $\bar{\sigma}$.

As strand separation occurs, daughter strand synthesis is catalyzed via enzymes known as DNA replicases. However, due to errors in the base pairing process, $\sigma$ is not necessarily paired with $\bar{\sigma}$. Rather, once cell division is finished, the original $\sigma$ is paired with some $\sigma^{\prime}$, and similarly for $\bar{\sigma}$.

Each genome $\{\sigma, \bar{\sigma}\}$ has a characteristic replication mismatch probability $\epsilon_{\{\sigma, \bar{\sigma}\}}$ (a base-pair-independent mismatch probability is certainly a simplification, but it is an initial

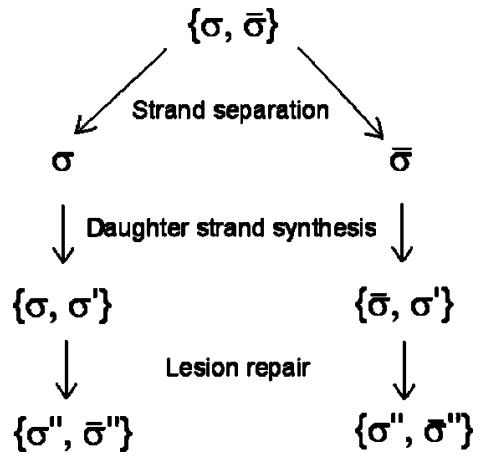

FIG. 2. The three stages of DNA replication.

starting point). Different genomes may have different replication fidelities, due to various replication error correction mechanisms which may or may not be functioning. For example, in Escherichia coli, the DNA replicase Pol III has a built-in proofreading mechanism which excises mismatched bases in the daughter strand [16]. In addition, in many prokaryotes and eukaryotes, DNA daughter strand synthesis is followed by mismatch repair [16], which can distinguish between the parent and daughter strands, thereby allowing the proper repair of mismatches. All such repair mechanisms are gathered within $\epsilon_{\{\sigma, \bar{\sigma}\}}$ in our model.

In the final stage, DNA replication and cell division is complete, and the parent and daughter strands have become indistinguishable. Remaining mismatches in the daughter cells' DNA are eliminated by various maintenance enzymes, which recognize and repair the lesions caused by mismatched base pairs. However, because it is impossible to determine which strand has the incorrect base, the mismatch is correctly repaired with probability $1 / 2$. The result is that the $\sigma, \sigma^{\prime}$ pair is converted to some $\sigma^{\prime \prime}, \bar{\sigma}^{\prime \prime}$, giving the DNA molecule $\left\{\sigma^{\prime \prime}, \bar{\sigma}^{\prime \prime}\right\}$. A similar process happens for the parent $\bar{\sigma}$ strand.

We wish to derive the probability that a given parent strand $\sigma$ produces $\left\{\sigma^{\prime \prime}, \bar{\sigma}^{\prime \prime}\right\}$ in the daughter cell. Let us denote this probability by $p\left(\sigma,\left\{\sigma^{\prime \prime}, \bar{\sigma}^{\prime \prime}\right\}\right)$. Also, let $p\left(\sigma, \sigma^{\prime}\right)$ denote the probability that $\sigma$ is paired with $\sigma^{\prime}$ during daughter strand synthesis, and let $p\left[\left(\sigma, \sigma^{\prime}\right),\left(\sigma^{\prime \prime}, \bar{\sigma}^{\prime \prime}\right)\right]$ be the probability that $\sigma \rightarrow \sigma^{\prime \prime}, \sigma^{\prime} \rightarrow \bar{\sigma}^{\prime \prime}$ during post-replicative lesion repair. Then we have, assuming $\sigma^{\prime \prime} \neq \bar{\sigma}^{\prime \prime}$, that

$$
\begin{aligned}
p\left(\sigma,\left\{\sigma^{\prime \prime}, \bar{\sigma}^{\prime \prime}\right\}\right)= & \sum_{\sigma^{\prime}} p\left(\sigma, \sigma^{\prime}\right)\left\{p\left[\left(\sigma, \sigma^{\prime}\right),\left(\sigma^{\prime \prime}, \bar{\sigma}^{\prime \prime}\right)\right]\right. \\
& \left.+p\left[\left(\sigma, \sigma^{\prime}\right),\left(\bar{\sigma}^{\prime \prime}, \sigma^{\prime \prime}\right)\right]\right\} \\
= & \sum_{\sigma^{\prime}} p\left(\sigma, \sigma^{\prime}\right) p\left[\left(\sigma, \sigma^{\prime}\right),\left(\sigma^{\prime \prime}, \bar{\sigma}^{\prime}\right)\right] \\
& +\sum_{\sigma^{\prime}} p\left(\sigma, \sigma^{\prime}\right) p\left[\left(\sigma, \sigma^{\prime}\right),\left(\bar{\sigma}^{\prime \prime}, \sigma^{\prime \prime}\right)\right] .
\end{aligned}
$$

If $\sigma^{\prime \prime}=\bar{\sigma}^{\prime \prime}$, then only one of the sums is kept.

We now proceed to compute $\Sigma_{\sigma^{\prime}} p\left(\sigma, \sigma^{\prime}\right) p\left[\left(\sigma, \sigma^{\prime}\right),\left(\sigma^{\prime \prime}, \bar{\sigma}^{\prime \prime}\right)\right]$. Write $\quad \sigma=b_{1} \cdots b_{L}, \quad \sigma^{\prime}$ $=b_{1}^{\prime} \cdots b_{L}^{\prime}$, and $\sigma^{\prime \prime}=b_{1}^{\prime \prime} \cdots b_{L}^{\prime \prime}$. Let $l \equiv D_{H}\left(\sigma, \sigma^{\prime \prime}\right)$. Let us consider some $i$ for which $b_{i}=b_{i}^{\prime \prime}$. Then $b_{L-i+1}^{\prime}$ can take on any 
value, for if $b_{L-i+1}^{\prime}=\bar{b}_{i}^{\prime \prime}$, then no repair is necessary, and we obtain $b_{i} \rightarrow\left(b_{i}^{\prime \prime}, \bar{b}_{i}^{\prime \prime}\right)$. If $b_{L-i+1}^{\prime} \neq \bar{b}_{i}^{\prime \prime}$, then repair is necessary, and with probability $1 / 2$ it is $b_{L-i+1}^{\prime}$ that is repaired to $\bar{b}_{i}^{\prime \prime}$, giving once again that $b_{i} \rightarrow\left(b_{i}^{\prime \prime}, \bar{b}_{i}^{\prime \prime}\right)$. So, let us now consider some $i$ for which $b_{i} \neq b_{i}^{\prime \prime}$. Then $b_{L-i+1}^{\prime}$ must be equal to $\bar{b}_{i}^{\prime \prime}$. Otherwise, if $b_{L-i+1}^{\prime}=\bar{b}_{i} \neq \bar{b}_{i}^{\prime \prime}$, then no lesion repair occurs, and we get $b_{i} \rightarrow\left(b_{i}, \bar{b}_{i}\right) \neq\left(b_{i}^{\prime \prime}, \bar{b}_{i}^{\prime \prime}\right)$. If $b_{L-i+1}^{\prime} \neq \bar{b}_{i}$, then $b_{L-i+1}^{\prime}$ is repaired with probability $1 / 2$ to $\bar{b}_{i}$, or $b_{i}$ is repaired with probability $1 / 2$ to $\bar{b}_{L-i+1}^{\prime}$. Thus, either $b_{i} \rightarrow\left(b_{i}, \bar{b}_{i}\right) \neq\left(b_{i}^{\prime \prime}, \bar{b}_{i}^{\prime \prime}\right)$, or $b_{i} \rightarrow\left(\bar{b}_{L-i+1}^{\prime}, b_{L-i+1}^{\prime}\right) \neq\left(b_{i}^{\prime \prime}, \bar{b}_{i}^{\prime \prime}\right)$, and so the corresponding $\sigma^{\prime}$ does not contribute to the sum $\Sigma_{\sigma^{\prime}} p\left(\sigma, \sigma^{\prime}\right) p\left[\left(\sigma, \sigma^{\prime}\right),\left(\sigma^{\prime \prime}, \bar{\sigma}^{\prime \prime}\right)\right], \quad$ since $p\left[\left(\sigma, \sigma^{\prime}\right),\left(\sigma^{\prime \prime}, \bar{\sigma}^{\prime \prime}\right)\right]$ $=0$.

Our analysis allows us to perform the sum, assuming a probability $\epsilon_{\{\sigma, \bar{\sigma}\}}$ of a mismatch. For a given $\sigma^{\prime}$, let $l^{\prime}$ denote the number of sites among the $L-l$ sites which are equal in $\sigma$ and $\sigma^{\prime \prime}$ for which $b_{L-i+1}^{\prime} \neq \bar{b}_{i}^{\prime \prime}$. There are $\left(\begin{array}{c}L-l \\ l^{\prime}\end{array}\right)(2 S-1)^{l^{\prime}}$ ways of choosing such a $\sigma^{\prime}$. The probability $p\left(\sigma, \sigma^{\prime}\right)$ is equal to $[\epsilon\{\sigma, \bar{\sigma}\} /(2 S-1)]^{I^{\prime}+l}\left(1-\epsilon_{\{\sigma, \bar{\sigma}\}}\right)^{L-l-l^{\prime}}$. The probability $p\left[\left(\sigma, \sigma^{\prime}\right),\left(\sigma^{\prime \prime}, \bar{\sigma}^{\prime \prime}\right)\right]$ is then $(1 / 2)^{l^{\prime}+l}$, so multiplying by the degeneracy in $l^{\prime}$ and summing over all $l^{\prime}$ gives

$$
\begin{aligned}
\sum_{\sigma^{\prime}} p\left(\sigma, \sigma^{\prime}\right) p\left[\left(\sigma, \sigma^{\prime}\right),\left(\sigma^{\prime \prime}, \bar{\sigma}^{\prime \prime}\right)\right] \\
=\sum_{l^{\prime}=0}^{L-l}\left(\begin{array}{c}
L-l \\
l^{\prime}
\end{array}\right)(2 S-1)^{l^{\prime}}\left(\frac{\epsilon_{\{\sigma, \bar{\sigma}\}}}{2 S-1}\right)^{l^{\prime}+l} \\
\times\left(1-\epsilon_{\{\sigma, \bar{\sigma}\}}\right)^{L-l-l^{\prime}}\left(\frac{1}{2}\right)^{l^{\prime}+l} \\
=\left(\frac{\epsilon_{\{\sigma, \bar{\sigma}\}} / 2}{2 S-1}\right)^{l}\left(1-\frac{\epsilon_{\left\{\sigma, \bar{\sigma}_{\}}\right\}}}{2}\right)^{L-l} .
\end{aligned}
$$

If we define $\bar{l}=D_{H}\left(\sigma, \bar{\sigma}^{\prime \prime}\right)$, then we obtain that $\Sigma_{\sigma^{\prime}} p\left(\sigma, \sigma^{\prime}\right) p\left[\left(\sigma, \sigma^{\prime}\right),\left(\bar{\sigma}^{\prime \prime}, \sigma^{\prime \prime}\right)\right]=\left[\left(\epsilon_{\{\sigma, \bar{\sigma}\}} / 2\right) /(2 S-1)\right]^{\bar{l}}(1$ $\left.-\epsilon_{\{\sigma, \bar{\sigma}\}} / 2\right)^{L-\bar{l}}$. Now, note that $D_{H}\left(b_{1} \cdots b_{L}, b_{1}^{\prime} \cdots b_{L}^{\prime}\right)$ $=D_{H}\left(\bar{b}_{L} \cdots \bar{b}_{1}, \bar{b}_{L}^{\prime} \cdots \bar{b}_{1}^{\prime}\right), \quad$ and $\quad D_{H}\left(b_{1} \cdots b_{L}, \bar{b}_{L}^{\prime} \cdots \bar{b}_{1}^{\prime}\right)$ $=D_{H}\left(\bar{b}_{L} \cdots \bar{b}_{1}, b_{1}^{\prime} \cdots b_{L}^{\prime}\right)$, so that $l=D_{H}\left(\sigma, \sigma^{\prime \prime}\right)=D_{H}\left(\bar{\sigma}, \bar{\sigma}^{\prime \prime}\right)$, and $\bar{l}=D_{H}\left(\sigma, \bar{\sigma}^{\prime \prime}\right)=D_{H}\left(\bar{\sigma}, \sigma^{\prime \prime}\right)$. Therefore, we obtain that

$$
p\left(\sigma,\left\{\sigma^{\prime \prime}, \bar{\sigma}^{\prime \prime}\right\}\right)=p\left(\bar{\sigma},\left\{\sigma^{\prime \prime}, \bar{\sigma}^{\prime \prime}\right\}\right)=\left\{\begin{array}{l}
\left(\frac{\epsilon_{\left\{\sigma, \bar{\sigma}_{\}} / 2\right.}}{2 S-1}\right)^{l}\left(1-\frac{\epsilon_{\{\sigma, \bar{\sigma}\}}}{2}\right)^{L-l}+\left(\frac{\epsilon_{\left\{\sigma, \bar{\sigma}_{\}}\right.} / 2}{2 S-1}\right)^{\bar{l}}\left(1-\frac{\epsilon_{\{\sigma, \bar{\sigma}\}}}{2}\right)^{L-\bar{l}} \text { for } \sigma^{\prime \prime} \neq \bar{\sigma}^{\prime \prime} \\
\left(\frac{\epsilon_{\{\sigma, \bar{\sigma}} / 2}{2 S-1}\right)^{l}\left(1-\frac{\epsilon_{\{\sigma, \bar{\sigma}\}}}{2}\right)^{L-l} \text { for } \sigma^{\prime \prime}=\bar{\sigma}^{\prime \prime}
\end{array}\right.
$$

\section{The quasispecies equations}

We are now ready to derive the quasispecies equations for semiconservative replication. We consider a population of unicellular, asexually replicating organisms. Let $n_{\{\sigma, \bar{\sigma}\}}$ denote the number of organisms with genome $\{\sigma, \bar{\sigma}\}$. We let $\kappa_{\{\sigma, \bar{\sigma}\}}$ denote the first-order growth rate constant of organisms with genome $\{\sigma, \bar{\sigma}\}$. Then from the replication mechanism illustrated in Fig. 2, we obtain the system of differential equations given by

$$
\begin{aligned}
\frac{d n_{\{\sigma, \bar{\sigma}\}}}{d t}= & -\kappa_{\{\sigma, \bar{\sigma}\}} n_{\{\sigma, \bar{\sigma}\}}+\sum_{\left\{\sigma^{\prime}, \bar{\sigma}^{\prime}\right\}} \kappa_{\left\{\sigma^{\prime}, \bar{\sigma}^{\prime}\right\}} n_{\left\{\sigma^{\prime}, \bar{\sigma}^{\prime}\right\}} \\
& \times\left[p\left(\sigma^{\prime},\{\sigma, \bar{\sigma}\}\right)+p\left(\bar{\sigma}^{\prime},\{\sigma, \bar{\sigma}\}\right)\right] .
\end{aligned}
$$

The first term is a death term which takes into account the destruction of the original genome during replication. The terms in the summation take into the account the production of $\{\sigma, \bar{\sigma}\}$ from both $\sigma^{\prime}$ and $\bar{\sigma}^{\prime}$.
We now define $n=\Sigma_{\{\sigma, \bar{\sigma}\}} n_{\{\sigma, \bar{\sigma}\}}$, and $x_{\{\sigma, \bar{\sigma}\}}=n_{\{\sigma, \bar{\sigma}\}} / n$. Reexpressed in terms of the population fractions $x_{\{\sigma, \bar{\sigma}\}}$, the dynamical equations become

$$
\begin{aligned}
\frac{d x_{\{\sigma, \bar{\sigma}\}}}{d t}= & \sum_{\left\{\sigma^{\prime}, \bar{\sigma}^{\prime}\right\}} \kappa_{\left\{\sigma^{\prime}, \bar{\sigma}^{\prime}\right\}} x_{\left\{\sigma^{\prime}, \bar{\sigma}^{\prime}\right\}} \\
& \times\left[p\left(\sigma^{\prime},\{\sigma, \bar{\sigma}\}\right)+p\left(\bar{\sigma}^{\prime},\{\sigma, \bar{\sigma}\}\right)\right] \\
& -\left[\kappa_{\{\sigma, \bar{\sigma}\}}+\bar{\kappa}(t)\right] x_{\{\sigma, \bar{\sigma}\}},
\end{aligned}
$$

where $\bar{\kappa}(t) \equiv \sum_{\{\sigma, \bar{\sigma}\}} \kappa_{\{\sigma, \bar{\sigma}\}} x_{\{\sigma, \bar{\sigma}\}}$ is the mean fitness of the population, which arises as a normalization term to ensure that the total population fraction remains 1 .

We now proceed to put these equations into a form which is more easily amenable to analysis than the above equations. To this end, we make the following definitions: (1) $\kappa_{\sigma}$ $\equiv \kappa_{\{\sigma, \bar{\sigma}\}}$, so that $\kappa_{\bar{\sigma}}=\kappa_{\sigma}$, (2) $\epsilon_{\sigma} \equiv \epsilon_{\{\sigma, \bar{\sigma}\}}$, so that $\epsilon_{\sigma}=\epsilon_{\bar{\sigma}}$. Fi- 
nally, $y_{\sigma} \equiv \frac{1}{2} x_{\{\sigma, \bar{\sigma}\}}$ if $\sigma \neq \bar{\sigma}$, and $y_{\sigma} \equiv x_{\{\sigma, \bar{\sigma}\}}$ if $\sigma=\bar{\sigma}$. Clearly, we also have that $y_{\sigma}=y_{\bar{\sigma}}$.

Now,

$$
\begin{aligned}
& \sum_{\left\{\sigma^{\prime}, \bar{\sigma}^{\prime}\right\}} \kappa_{\left\{\sigma^{\prime}, \bar{\sigma}^{\prime}\right\}} x_{\left\{\sigma^{\prime}, \bar{\sigma}^{\prime}\right\}}\left[p\left(\sigma^{\prime},\{\sigma, \bar{\sigma}\}\right)+p\left(\bar{\sigma}^{\prime},\{\sigma, \bar{\sigma}\}\right)\right] \\
& =\sum_{\left\{\sigma^{\prime}, \bar{\sigma}^{\prime}\right\}, \sigma^{\prime} \neq \bar{\sigma}^{\prime}} \kappa_{\left\{\sigma^{\prime}, \bar{\sigma}^{\prime}\right\}} x_{\left\{\sigma^{\prime}, \bar{\sigma}^{\prime}\right\}}\left[p\left(\sigma^{\prime},\{\sigma, \bar{\sigma}\}\right)+p\left(\bar{\sigma}^{\prime},\{\sigma, \bar{\sigma}\}\right)\right] \\
& \quad+\sum_{\left\{\sigma^{\prime}, \bar{\sigma}^{\prime}\right\}, \sigma^{\prime}=\bar{\sigma}^{\prime}} \kappa_{\left\{\sigma^{\prime}, \bar{\sigma}^{\prime}\right\}} x_{\left\{\sigma^{\prime}, \bar{\sigma}^{\prime}\right\}}\left[p\left(\sigma^{\prime},\{\sigma, \bar{\sigma}\}\right)\right. \\
& \left.\quad+p\left(\bar{\sigma}^{\prime},\{\sigma, \bar{\sigma}\}\right)\right], \\
& =\sum_{\left\{\sigma^{\prime}, \bar{\sigma}^{\prime}\right\}, \sigma^{\prime} \neq \bar{\sigma}^{\prime}} \kappa_{\sigma^{\prime}} x_{\left\{\sigma^{\prime}, \bar{\sigma}^{\prime}\right\}} p\left(\sigma^{\prime},\{\sigma, \bar{\sigma}\}\right) \\
& \quad+\sum_{\left\{\sigma^{\prime}, \bar{\sigma}^{\prime}\right\}, \sigma^{\prime} \neq \bar{\sigma}^{\prime}} \kappa_{\bar{\sigma}^{\prime}} x_{\left\{\sigma^{\prime}, \bar{\sigma}^{\prime}\right\}} p\left(\bar{\sigma}^{\prime},\{\sigma, \bar{\sigma}\}\right) \\
& \quad+\sum_{\left\{\sigma^{\prime}, \bar{\sigma}^{\prime}\right\}, \sigma^{\prime}=\bar{\sigma}^{\prime}} 2 \kappa_{\sigma^{\prime}} x_{\left\{\sigma^{\prime}, \bar{\sigma}^{\prime}\right\}} p\left(\sigma^{\prime},\{\sigma, \bar{\sigma}\}\right) \\
& =\sum_{\sigma^{\prime}, \sigma^{\prime} \neq \bar{\sigma}^{\prime}} \kappa_{\sigma^{\prime}} x_{\left\{\sigma^{\prime}, \bar{\sigma}^{\prime}\right\}} p\left(\sigma^{\prime},\{\sigma, \bar{\sigma}\}\right) \\
& \quad+2 \sum_{\sigma^{\prime}, \sigma^{\prime}=\bar{\sigma}^{\prime}} \kappa_{\sigma^{\prime}} x_{\left\{\sigma^{\prime}, \bar{\sigma}^{\prime}\right\}} p\left(\sigma^{\prime},\{\sigma, \bar{\sigma}\}\right) .
\end{aligned}
$$

Therefore, for $\sigma \neq \bar{\sigma}$,

$$
\begin{aligned}
\frac{d y_{\sigma}}{d t}= & \frac{1}{2} \frac{d x_{\{\sigma, \bar{\sigma}\}}}{d t} \\
= & \frac{1}{2} \sum_{\sigma^{\prime}, \sigma^{\prime} \neq \bar{\sigma}^{\prime}} 2 \kappa_{\sigma^{\prime}} y_{\sigma^{\prime}}\left[\left(\frac{\epsilon_{\sigma^{\prime}} / 2}{2 S-1}\right)^{D_{H}\left(\sigma, \sigma^{\prime}\right)}\right. \\
& \times\left(1-\frac{\epsilon_{\sigma^{\prime}}}{2}\right)^{L-D_{H}\left(\sigma, \sigma^{\prime}\right)}+\left(\frac{\epsilon_{\sigma^{\prime}} / 2}{2 S-1}\right)^{D_{H}\left(\bar{\sigma}, \sigma^{\prime}\right)} \\
& \left.\times\left(1-\frac{\epsilon_{\sigma^{\prime}}}{2}\right)^{L-D_{H}\left(\bar{\sigma}, \sigma^{\prime}\right)}\right] \\
& +\sum_{\sigma^{\prime}, \sigma^{\prime}=\bar{\sigma}^{\prime}} \kappa_{\sigma^{\prime}} y_{\sigma^{\prime}}\left[\left(\frac{\epsilon_{\sigma^{\prime}} / 2}{2 S-1}\right)^{D_{H}\left(\sigma, \sigma^{\prime}\right)}\right. \\
& \times\left(1-\frac{\epsilon_{\sigma^{\prime}}}{2}\right)^{L-D_{H}\left(\sigma, \sigma^{\prime}\right)}+\left(\frac{\epsilon_{\sigma^{\prime}} / 2}{2 S-1}\right)^{D_{H}\left(\bar{\sigma}, \sigma^{\prime}\right)} \\
& \left.\times\left(1-\frac{\epsilon_{\sigma^{\prime}}}{2}\right)^{L-D_{H}\left(\bar{\sigma}, \sigma^{\prime}\right)}\right]-\left[\kappa_{\sigma}+\bar{\kappa}(t)\right] y_{\sigma} \\
= & \sum_{\sigma^{\prime}} \kappa_{\sigma^{\prime}} y_{\sigma^{\prime}}\left[\left(\frac{\epsilon_{\sigma^{\prime}} / 2}{2 S-1}\right)^{D_{H}\left(\sigma, \sigma^{\prime}\right)}\left(1-\frac{\epsilon_{\sigma^{\prime}}}{2}\right)^{L-D_{H}\left(\sigma, \sigma^{\prime}\right)}\right. \\
& \left.+\left(\frac{\epsilon_{\sigma^{\prime}} / 2}{2 S-1}\right)^{D_{H}\left(\bar{\sigma}, \sigma^{\prime}\right)}\left(1-\frac{\epsilon_{\sigma^{\prime}}}{2}\right)^{L-D_{H}\left(\bar{\sigma}, \sigma^{\prime}\right)}\right] \\
& -\left[\kappa_{\sigma}+\bar{\kappa}(t)\right] y_{\sigma}
\end{aligned}
$$

$$
\begin{aligned}
= & \sum_{\sigma^{\prime}} \kappa_{\sigma^{\prime}} y_{\sigma^{\prime}}\left(\frac{\epsilon_{\sigma^{\prime}} / 2}{2 S-1}\right)^{D_{H}\left(\sigma, \sigma^{\prime}\right)} \\
& \times\left(1-\frac{\epsilon_{\sigma^{\prime}}}{2}\right)^{L-D_{H}\left(\sigma, \sigma^{\prime}\right)}+\sum_{\sigma^{\prime}} \kappa_{\bar{\sigma}^{\prime}} y_{\bar{\sigma}^{\prime}}\left[\left(\frac{\epsilon_{\bar{\sigma}^{\prime}} / 2}{2 S-1}\right)^{D_{H}\left(\sigma, \bar{\sigma}^{\prime}\right)}\right. \\
& \left.\times\left(1-\frac{\epsilon_{\bar{\sigma}^{\prime}}}{2}\right)^{L-D_{H}\left(\sigma, \bar{\sigma}^{\prime}\right)}\right]-\left[\kappa_{\sigma}+\bar{\kappa}(t)\right] y_{\sigma} \\
= & 2 \sum_{\sigma^{\prime}} \kappa_{\sigma^{\prime}} y_{\sigma^{\prime}}\left(\frac{\epsilon_{\sigma^{\prime}} / 2}{2 S-1}\right)^{D_{H}\left(\sigma, \sigma^{\prime}\right)}\left(1-\frac{\epsilon_{\sigma^{\prime}}}{2}\right)^{L-D_{H}\left(\sigma, \sigma^{\prime}\right)} \\
& -\left[\kappa_{\sigma}+\bar{\kappa}(t)\right] y_{\sigma} .
\end{aligned}
$$

For $\sigma=\bar{\sigma}$ we get

$$
\begin{aligned}
\frac{d y_{\sigma}}{d t}= & \frac{d x_{\{\sigma, \bar{\sigma}\}}}{d t} \\
= & \sum_{\sigma^{\prime}, \sigma^{\prime} \neq \bar{\sigma}^{\prime}} 2 \kappa_{\sigma^{\prime}} y_{\sigma^{\prime}} p\left(\sigma^{\prime},\{\sigma, \bar{\sigma}\}\right) \\
& +2 \sum_{\sigma^{\prime}, \sigma^{\prime}=\bar{\sigma}^{\prime}} \kappa_{\sigma^{\prime}} y_{\sigma^{\prime}} p\left(\sigma^{\prime},\{\sigma, \bar{\sigma}\}\right)-\left[\kappa_{\sigma}+\bar{\kappa}(t)\right] y_{\sigma} \\
= & 2 \sum_{\sigma^{\prime}} \kappa_{\sigma^{\prime}} y_{\sigma^{\prime}}\left(\frac{\epsilon_{\sigma^{\prime}} / 2}{2 S-1}\right)^{D_{H}\left(\sigma, \sigma^{\prime}\right)}\left(1-\frac{\epsilon_{\sigma^{\prime}}}{2}\right)^{L-D_{H}\left(\sigma, \sigma^{\prime}\right)} \\
& -\left[\kappa_{\sigma}+\bar{\kappa}(t)\right] y_{\sigma^{\prime}} .
\end{aligned}
$$

Since we obtain the same set of equations for palindromic and non-palindromic molecules, the final form of our quasispecies equations becomes

$$
\begin{aligned}
\frac{d y_{\sigma}}{d t}= & 2 \sum_{\sigma^{\prime}} \kappa_{\sigma^{\prime}} y_{\sigma^{\prime}}\left(\frac{\epsilon_{\sigma^{\prime}} / 2}{2 S-1}\right)^{D_{H}\left(\sigma, \sigma^{\prime}\right)}\left(1-\frac{\epsilon_{\sigma^{\prime}}}{2}\right)^{L-D_{H}\left(\sigma, \sigma^{\prime}\right)} \\
& -\left[\kappa_{\sigma}+\bar{\kappa}(t)\right] y_{\sigma} .
\end{aligned}
$$

It is readily shown that $\bar{\kappa}(t)=\Sigma_{\sigma} \kappa_{\sigma} y_{\sigma}$. It is also readily shown that $y_{\sigma}=y_{\bar{\sigma}}$ for all $\sigma$ implies that $d y_{\sigma} / d t=d y_{\bar{\sigma}} / d t$ for all $\sigma$, and so $y_{\sigma}=y_{\bar{\sigma}}$ is preserved by the evolution.

As a final note in this section, we can express the above system of equations in vector form as follows: We define $\vec{y}$ $=\left(y_{\sigma}\right)$, so $\vec{y}$ is simply the vector of population fractions. We define $\vec{\kappa}=\left(\kappa_{\sigma}\right)$, so $\vec{\kappa}$ is simply the vector of growth rate constants. We define

$$
\mathbf{A}=\left\{\left[A_{\sigma \sigma^{\prime}}=\kappa_{\sigma^{\prime}}\left(\frac{\epsilon_{\sigma^{\prime}} / 2}{2 S-1}\right)^{D_{H}\left(\sigma, \sigma^{\prime}\right)}\left(1-\frac{\epsilon_{\sigma^{\prime}}}{2}\right)^{L-D_{H}\left(\sigma, \sigma^{\prime}\right)}\right]\right\}
$$

to be the matrix of first-order mutation rate constants. Finally, we define $\mathbf{K}=\left[\left(K_{\sigma \sigma^{\prime}}=\kappa_{\sigma} \delta_{\sigma \sigma^{\prime}}\right)\right]$ to be the diagonal matrix of first-order growth rate constants. Putting everything together, we obtain the vector form of the quasispecies equations 
TABLE I. Comparison of quasispecies equilibrium between conservative and semiconservative replication. It should be noted that $\bar{\kappa}(t=\infty)$ is simply the equilibrium mean fitness of the population.

\begin{tabular}{ccc}
\hline \hline Parameter & Conservative & Semiconservative \\
\hline$\mu_{\text {crit }}$ & $\ln k$ & $2 \ln [2 /(1+1 / k)]$ \\
$x_{\text {master }}\left(\mu<\mu_{\text {crit }}\right)$ & $\left(k e^{-\mu}-1\right) /(k-1)$ & $\left(k\left(2 e^{-\mu / 2}-1\right)-1\right) /(k-1)$ \\
$\bar{\kappa}(t=\infty)\left(\mu<\mu_{\text {crit }}\right)$ & $k e^{-\mu}$ & $\left.k) 2 e^{-\mu / 2}-1\right)$ \\
$\langle l\rangle\left(\mu<\mu_{\text {crit }}\right)$ & $\mu k e^{-\mu} /\left(k e^{-\mu}-1\right)$ & $\mu k\left(2 e^{-\mu / 2}-1\right) /\left(k\left(2 e^{-\mu / 2}-1\right)-1\right)$ \\
\hline
\end{tabular}

$$
\frac{d \vec{y}}{d t}=(2 \mathbf{A}-\mathbf{K}) \vec{y}-(\vec{\kappa} \cdot \vec{y}) \vec{y} .
$$

Presumably, for a static landscape, the system of equations converges to the equilibrium distribution given by the eigenvector corresponding to the largest eigenvalue of the matrix $2 \mathbf{A}-\mathbf{K}$.

\section{THE SINGLE FITNESS PEAK}

\section{A. Overview and analytical results}

In the single fitness peak model, there exists a unique, master genome $\left\{\sigma_{0}, \bar{\sigma}_{0}\right\}$ with fitness $k>1$, with all other genomes having fitness 1 . Our fitness landscape is therefore given by $\kappa_{\sigma_{0}}=\kappa_{\bar{\sigma}_{0}}=k$, while $\kappa_{\sigma}=1$ for $\sigma \neq \sigma_{0}, \bar{\sigma}_{0}$. We also assume that $\epsilon_{\sigma}$ is independent of $\sigma$, so that $\epsilon_{\sigma}=\epsilon$. For this landscape, we wish to obtain the equilibrium behavior of the system of differential equations given by Eq. (15).

For the case of conservative replication, the single fitness peak model may be solved by first grouping the genomes into Hamming classes [3]. Specifically, given the master sequence $\sigma_{0}$, we may define $C_{H}(l)=\left\{\sigma \mid D_{H}\left(\sigma, \sigma_{0}\right)=l\right\}$. If $x_{\sigma}$ denotes the population fraction with genome $\sigma$, then we define $z_{l}=\Sigma_{\sigma \in C_{H}(l)} x_{\sigma}$. The quasispecies equations are then reexpressed in terms of the $z_{l}$, and the equilibrium equations may be readily solved in the limit of infinite sequence length, since the backmutation terms become negligible. The result is

$$
\frac{d z_{l}}{d t}=e^{-\mu} \sum_{l_{1}=0}^{l} \frac{1}{l_{1} !} \kappa_{l-l_{1}} \mu^{l_{1}} z_{l-l_{1}}-\bar{\kappa}(t) z_{l},
$$

where $\kappa_{l}=k$ for $l=0$, and 1 for $l>0, \bar{\kappa}(t)=(k-1) z_{0}+1$, and $\mu \equiv L \epsilon$ in the limit $L \rightarrow \infty$.

For the case of semiconservative replication, the single fitness peak model for double-stranded genomes becomes an effectively two fitness peak model. Thus, it is not possible to directly group the genomes into Hamming classes. Nevertheless, the single fitness peak for double-stranded genomes is solvable. The details of the solution, which are fairly involved, may be found in Appendix A. The final result, however, is simple to understand. In the limit of infinite sequence length, $\sigma_{0}$ and $\bar{\sigma}_{0}$ become infinitely separated. Therefore, locally around $\sigma_{0}, \bar{\sigma}_{0}$ we have an effectively single fitness peak model. We may therefore exploit the local symmetry of the landscape and define Hamming classes around $\sigma_{0}$ and $\bar{\sigma}_{0}$.
Thus, $C_{H}\left(\sigma_{0} ; l\right) \equiv\left\{\sigma \mid D_{H}\left(\sigma, \sigma_{0}\right)=l\right\}$, and similarly for $\bar{\sigma}_{0}$. We may then define $w_{l}=\Sigma_{\sigma \in C_{H}\left(\sigma_{0} ; l\right)} y_{\sigma}$, and $\bar{w}_{l}$ may be defined similarly with respect to $\bar{\sigma}_{0}$. However, by symmetry of the landscape we have $w_{l}=\bar{w}_{l}$, and so need only consider the dynamics of the $w_{l}$. In Appendix A, we show that when expressed in terms of the $w_{l}$, the quasispecies equations become

$$
\frac{d w_{l}}{d t}=2 e^{-\mu / 2} \sum_{l_{1}=0}^{l} \frac{1}{l_{1} !}\left(\frac{\mu}{2}\right)^{l_{1}} \kappa_{l-l_{1}} w_{l-l_{1}}-\left[\kappa_{l}+\bar{\kappa}(t)\right] w_{l} .
$$

In this case, $\bar{\kappa}(t)=2(k-1) w_{0}+1$. The reason for this is that $w_{0}$ is only the fraction of the population on the fitness peak at $\sigma_{0}$. By the way we defined our $y_{\sigma}$, the total fraction of viable organisms is given by $w_{0}+\bar{w}_{0}=2 w_{0}$.

We begin the solution of the infinite sequence length equations by solving for the equilibrium value of $w_{0}$. We have

$$
\frac{d w_{0}}{d t}=2 k e^{-\mu / 2} w_{0}-[k+\bar{\kappa}(t)] w_{0}
$$

which admits the solutions $w_{0}=0,\left[k\left(2 e^{-\mu / 2}-1\right)-1\right] / 2(k-1)$. Multiplying by 2, we get the equilibrium solution for $x_{\left\{\sigma_{0}, \bar{\sigma}_{0}\right\}}$ of 0 or $\left[k\left(2 e^{-\mu / 2}-1\right)-1\right] /(k-1)$. To determine the domain of validity of these solutions, we note that we want $w_{0}=1 / 2$ for $\mu=0$. That is, when replication is perfect, then the population resides entirely on the fitness peak $\left\{\sigma_{0}, \bar{\sigma}_{0}\right\}$. We must also have $w_{0} \geqslant 0$, which holds as long as $k\left(2 e^{-\mu / 2}-1\right)-1$ $\geqslant 0 \Rightarrow \mu \leqslant \mu_{\text {crit }} \equiv 2 \ln [2 /(1+1 / k)]$. Therefore, by continuity, we have that for $\mu \leqslant \mu_{\text {crit }}$, the equilibrium solution is $w_{0}$ $=\left[k\left(2 e^{-\mu / 2}-1\right)-1\right] / 2(k-1)$. For $\mu>\mu_{\text {crit }}$, the equilibrium solution becomes $w_{0}=0$. The transition between these two solution regimes is known as the error catastrophe.

In dealing with conservative replication, another parameter of interest which we consider is the localization length, defined as $\langle l\rangle=\sum_{l=1}^{\infty} l z_{l}$, where $z_{l}$ denotes the population fraction at localization length $l$ from the master sequence. We wish to extend the definition of localization length to our model. The complication here is that in the limit of infinite sequence length, the Hamming distances $l$ and $\bar{l}$ to $\sigma_{0}$ and $\bar{\sigma}_{0}$ (respectively) cannot be simultaneously finite. However, as mentioned previously, the fraction of the population at a Hamming distance $l$ from $\sigma_{0}$, given by $w_{l}$, is equal to the fraction of the population at a Hamming distance $l$ from $\bar{\sigma}_{0}$, given by $\bar{w}_{l}$. Therefore, an appropriate definition for the 


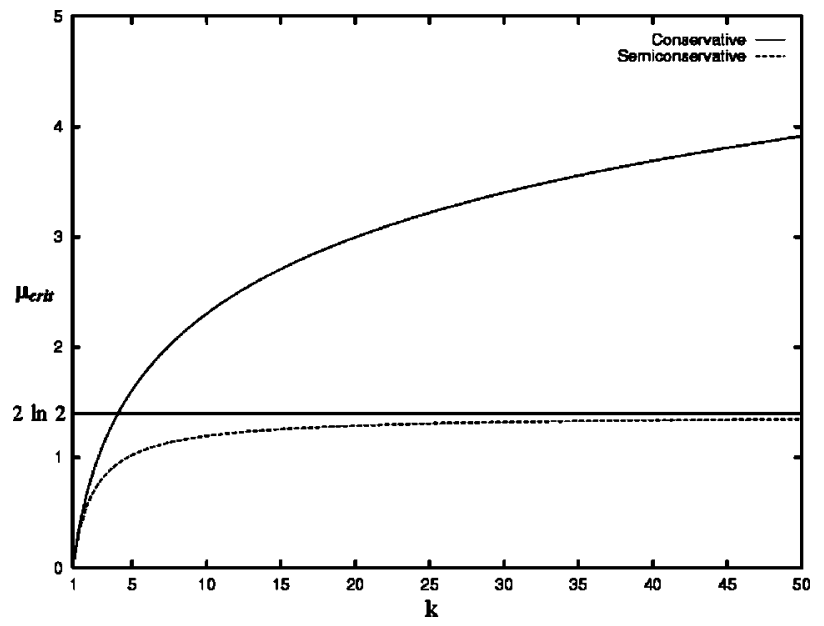

FIG. 3. Plot of $\mu_{\text {crit }}$ versus $k$ for both conservative and semiconservative replication. Units are dimensionless.

localization length is to define $\langle l\rangle=\sum_{l=1}^{\infty} 2 l w_{l}$. We may compute $\langle l\rangle$ by using a technique similar to the one developed in Ref. [14]. Briefly, a differential equation for the time evolution of $\langle l\rangle$ is derived from the evolution equations for the $w_{l}$. The result is

$$
\frac{d\langle l\rangle}{d t}=\langle l\rangle[1-\bar{\kappa}(t)]+\mu \bar{\kappa}(t)
$$

giving at equilibrium that

$$
\langle l\rangle=\mu \frac{\bar{\kappa}(t=\infty)}{\bar{\kappa}(t=\infty)-1}=\mu \frac{k\left(2 e^{-\mu / 2}-1\right)}{k\left(2 e^{-\mu / 2}-1\right)-1} .
$$

Note that the localization length is finite for $\mu<\mu_{\text {crit }}$, but diverges at the error catastrophe. For convenience, Table I illustrates the difference between conservative and semiconservative replication.

Figure 3 shows a plot of $\mu_{\text {crit }}$ versus $k$ for both the conservative and semiconservative cases. Figure 4 shows a plot of $\bar{\kappa}(t=\infty)$ versus $\mu$ for $k=10$ for both the conservative and

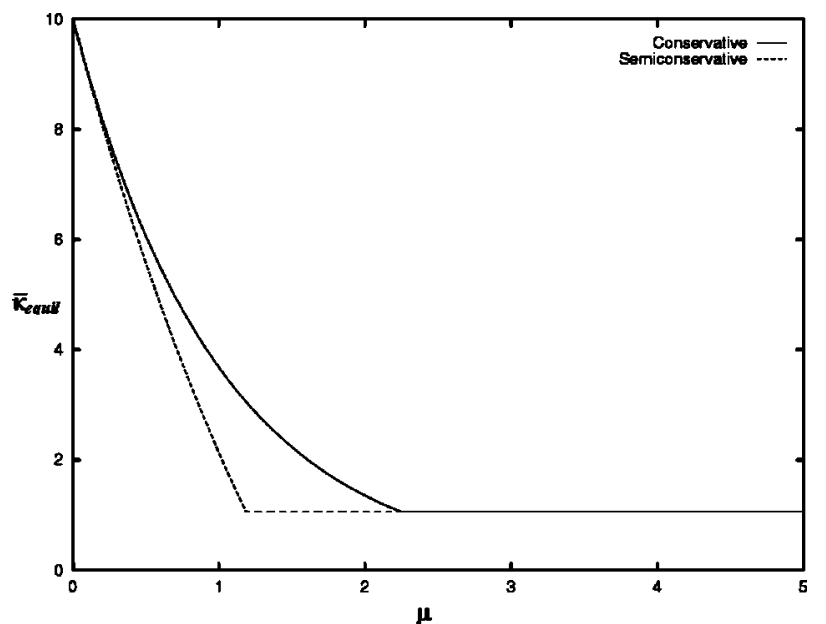

FIG. 4. Plot of $\bar{\kappa}(t=\infty)$ versus $\mu$ for $k=10$. Units are dimensionless.

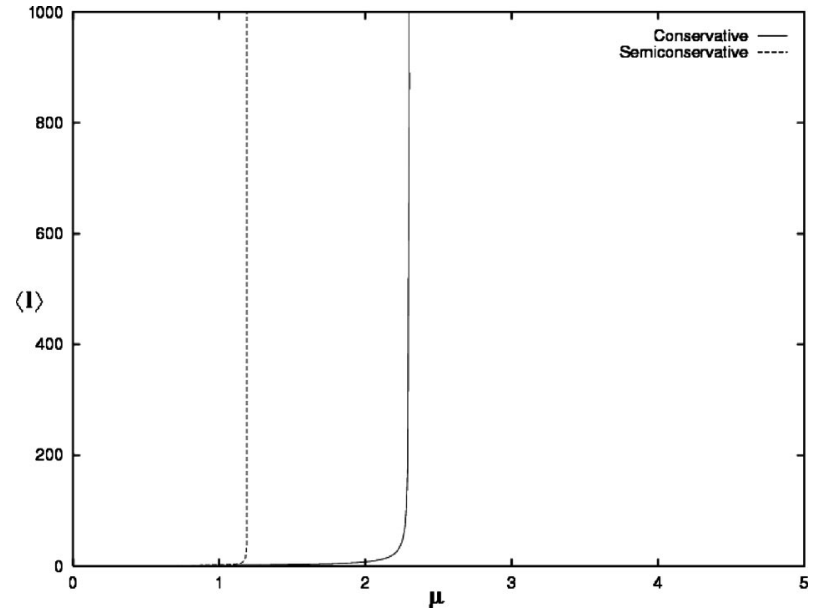

FIG. 5. Plot of $\langle l\rangle$ versus $\mu$ for $k=10$. Units are dimensionless.

semiconservative cases. Finally, Fig. 5 shows a plot of $\langle l\rangle$ versus $\mu$ for $k=10$ for both the conservative and semiconservative cases.

\section{B. Stochastic simulation of the semiconservative equations}

In order to complement the analytical work derived in this paper, we present stochastic simulations of semiconservatively replicating organisms, which numerically confirm the predicted location of the error catastrophe. The results of one of these simulations is shown in Fig. 6.

The results shown in Fig. 6 were obtained using a constant population size of 10000 organisms with genome lengths of 101 base pairs, using an alphabet size of $2 S=4$ (to correspond with the alphabet size of DNA). The master sequence replicates at each time step with probability $p_{R,\left\{\sigma_{0}, \bar{\sigma}_{0}\right\}}=1$; all other sequences replicate with

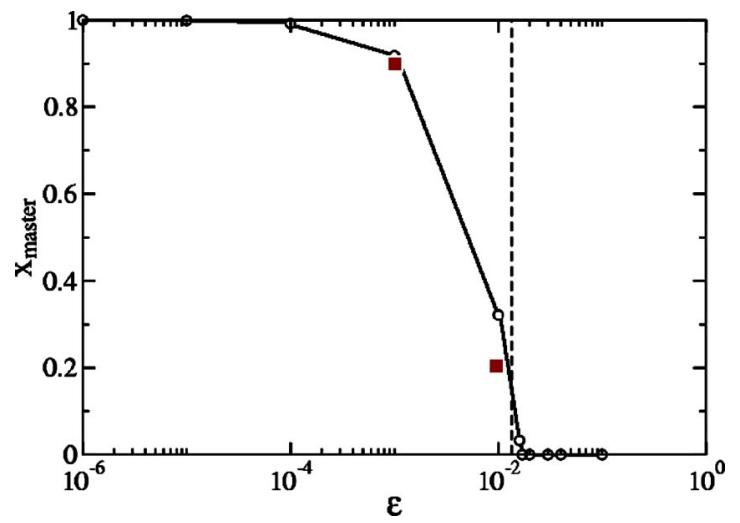

FIG. 6. Error catastrophe in a stochastic simulation of a finite population of 10000 semiconservatively replicating organisms. Where the analytical results differ noticeably from the numerical results, the analytical results are marked with filled squares. This discrepancy is simply a consequence of the finite size of the population and does not reflect a problem with the differential equations themselves, which assume an infinite population. Units in this figure are dimensionless. 
$p_{R,\{\sigma, \bar{\sigma}\} \neq\left\{\sigma_{0}, \bar{\sigma}_{0}\right\}}=0.01$, giving $\kappa_{\left\{\sigma_{0}, \bar{\sigma}_{0}\right\}}=100$, and $\kappa_{\{\sigma, \bar{\sigma}\} \neq\left\{\sigma_{0}, \bar{\sigma}_{0}\right\}}$ $=1$. By iterating a sufficient number of times, it is possible to compute the equilibrium value of the master sequence fraction $x_{\text {master }}$ for various values of $\epsilon$. The predicted $\epsilon_{\text {crit }}$ for the above parameters is indicated in Fig. 6 as a dashed line. Note the good agreement between the theoretical prediction of the error catastrophe and the numerical results.

\section{DISCUSSION}

The key difference between conservative replication and semiconservative replication is the destruction of the parent genome in the semiconservative case, as opposed to its preservation in the conservative case. This is captured by the functions $e^{-\mu}$ versus $2 e^{-\mu / 2}-1$ in the formulas given in Table I. For conservative replication, $e^{-\mu}$ is simply the probability of correct replication. This probability is always positive, and so, by making $k$ sufficiently large, it is possible to guarantee that the effective growth rate $k e^{-\mu}$ of the master sequence stays above the growth rate of 1 for the unviable sequences. For semiconservative replication, the probability that each strand is matched with its proper complementary strand is $e^{-\mu / 2}$. Therefore, since there are two parent strands, and the parent genome is destroyed during replication, we have the factor $2 e^{-\mu / 2}-1$, yielding an effective growth rate of $k\left(2 e^{-\mu / 2}-1\right)$. However, $2 e^{-\mu / 2}-1$ is only positive when $e^{-\mu / 2}>1 / 2$, or when $\mu<2 \ln 2$. When the probability of correct daughter strand synthesis drops below $1 / 2$, then the rate of production of viable genomes no longer exceeds the rate of destruction. The result is that replicating faster simply increases the rate of destruction of viable organisms, and therefore does not avoid the error catastrophe. This of course implies that at sufficiently high error rates, faster replicating cells will die more quickly than more slowly replicating cells. Thus, in contrast to the conservative quasispecies equations, the semiconservative equations provide a mathematical basis for explaining the efficacy of mutagens as chemotherapeutic agents.

The semiconservative quasispecies formalism may be naturally extended to more sophisticated models with more than one gene. In this paper, we focused on the single fitness peak model, in which the genome consists of a single, "viability," or "reproductive rate," "gene," and the replication error probability is genome independent.

As an example, we may incorporate mismatch repair into the semiconservative, quasispecies formalism. As with the conservative case $[13,14]$, we consider a two-gene model, in which one gene codes for viability, and the other codes for repair. Thus, a given genome $\{\sigma, \bar{\sigma}\}$ may be written as $\left\{\sigma_{\text {via }} \sigma_{\text {rep }}, \bar{\sigma}_{\text {rep }} \bar{\sigma}_{\text {via }}\right\}$. As was done in Refs. [13,14], we may assume a single-fitness peak in both the viability and repair genes, so that there exist "master" sequences $\sigma_{\text {via }, 0}, \bar{\sigma}_{\text {via }, 0}$, and $\sigma_{\text {rep }, 0}, \bar{\sigma}_{\text {rep }, 0}$ for both viability and repair, respectively. In the single-stranded formulation of the semiconservative model, a given $\sigma$ has a first-order growth rate $k>1$ if $\sigma$ $=\sigma_{\text {via }, 0} \sigma_{\text {rep }}$ or $\bar{\sigma}_{\text {rep }} \bar{\sigma}_{\text {via }, 0}$. The growth rate constant is 1 otherwise. Furthermore, $\sigma$ has a functioning mismatch repair system with failure probability $\epsilon_{r}$ if $\sigma=\sigma_{\text {via }} \sigma_{\text {rep }, 0}$ or $\bar{\sigma}_{\text {rep }, 0} \bar{\sigma}_{\text {via }}$. Otherwise, mismatch repair is inactivated.
While we leave the solution of this two-gene model for future work, we may nevertheless compute the location of the repair catastrophe. As with the case for conservative replication, the repair catastrophe occurs when the effective growth rate constant of viable repairers drops below the growth rate constant of viable nonrepairers. For viable repairers, the effective growth rate constant is $k\left(2 e^{-\epsilon_{r} \mu / 2}-1\right)$. We have for the nonrepairers an effective growth rate constant of viable organisms given by $k\left(2 e^{-\left(L_{\mathrm{via}} / L\right) \mu / 2}-1\right)$. The factor of $L_{\mathrm{via}} / L$ arises because in dealing with the overall growth rate of the mutators, we are only concerned with the production of viable organisms. The repairer gene does not need to be correctly replicated. The repair catastrophe then occurs when $k\left(2 e^{-\epsilon_{r} \mu / 2}-1\right)=k\left(2 e^{-\left(L_{\mathrm{via}} / L\right) \mu / 2}-1\right)$, or when $\epsilon_{r}$ $=L_{\mathrm{via}} / L$. Interestingly, this result is unchanged from the point mutation, conservative result in Ref. [13], or the full solution, conservative result in Ref. [14].

\section{CONCLUSIONS}

This paper extended the quasispecies formalism to include the case of semiconservative replication, in order to allow for the more realistic modeling of the evolutionary dynamics of DNA-based life. While we believe that this extension is an important first step in moving away from Eigen's original RNA-based model, much more work remains to be done. Our model is currently most directly applicable to prokaryotic genomes, which generally consist of a single, circular DNA molecule. This asssumes that the primary source of mutations in prokaryotes are point mutations. At high mutation rates (induced, for example, by nucleoside analogues), this may indeed be the case. However, a proper modeling of evolutionary dynamics will need to include other effects such as recombination, sex, insertions, deletions, gene duplications, and transposition (which is believed to play an important role in the spread of antibiotic drug resistance). Furthermore, in order to properly model eukaryotic genomes, it will be necessary to extend the quasispecies equations to genomes consisting of multiple chromosomes.

After deriving the quasispecies equations for semiconservative systems, we proceeded to solve them for the simplest landscape, that of the static single fitness peak. As with conservative replication, the solution of the single fitness peak yielded two regimes: A viable regime, where the population is localized about the "master" genome and an unviable regime, where the population is delocalized over the genome space. The transition between the two regimes is known as the error catastrophe.

The main difference between conservative and semiconservative replication is that for conservative replication, it is possible to push the error catastrophe to arbitrarily high replication error rates by increasing the growth rate constant of the master genome. In semiconservative replication, on the other hand, the probability of correct replication must always be greater than $1 / 2$, in order to avoid the error catastrophe. Semiconservative replication is therefore considerably less robust to the effect of mutagens than conservative replication. Furthermore, as pointed out in the Discussion, the existence of a lower bound to semiconservative replication fi- 


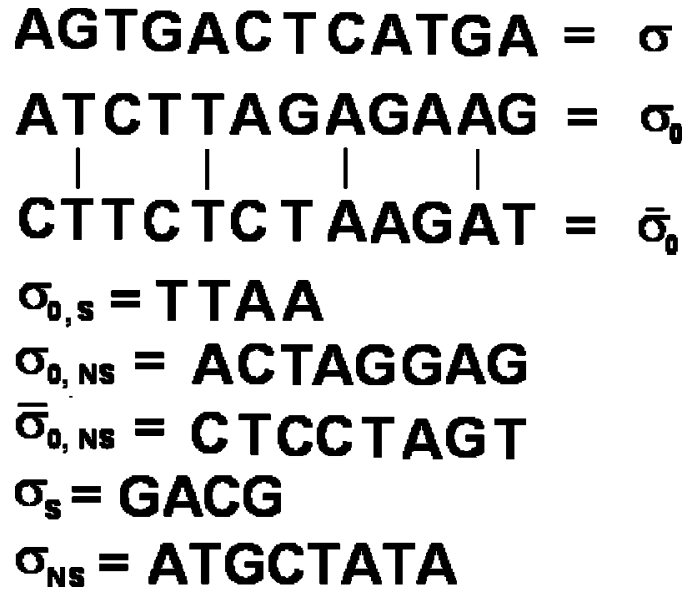

FIG. 7. Illustration of sequence decomposition into $\sigma_{S}$ and $\sigma_{N S}$ components.

delity explains why above a threshold mutation rate, mutagenic agents kill more rapidly replicating cells faster than more slowly replicating cells.

\section{ACKNOWLEDGMENTS}

E.T. would like to thank the National Institutes of Health for funding this research. He would also like to thank his instructors in the Israel Defense Forces' combat medic course for discussions about the effects of mutagens on slow and fast replicating cells. E.J.D. would like to thank the Howard Hughes Medical Institute for financial support.

\section{APPENDIX: SOLUTION OF THE STATIC SINGLE FITNESS PEAK MODEL FOR SEMICONSERVATIVE REPLICATION}

\section{Finite genome size equations}

To begin, let us define the internal Hamming distance $l_{I}$ $=D_{H}\left(\sigma_{0}, \bar{\sigma}_{0}\right)$. Also, let $\sigma_{0, S}$ denote the subsequence of bases where $\sigma_{0}$ and $\bar{\sigma}_{0}$ are identical, and $\sigma_{0, N S}$ and $\bar{\sigma}_{0, N S}$ denote the subsequences of bases in $\sigma_{0}$ and $\bar{\sigma}_{0}$, respectively, where they differ. Then given some gene sequence $\sigma$, we can break it up into two subsequences $\sigma_{S}$ and $\sigma_{N S} . \sigma_{S}$ denotes the subsequence of bases in $\sigma$ corresponding to the subsequence of bases where $\sigma_{0}, \bar{\sigma}_{0}$ are identical. $\sigma_{N S}$ denotes the subsequence of remaining bases. This is illustrated in Fig. 7.

Given some gene sequence $\sigma$, we can then characterize it by the following numbers: (1) $l_{S} \equiv D_{H}\left(\sigma_{S}, \sigma_{0, S}\right)$, (2) $l_{N S}$ $\equiv D_{H}\left(\sigma_{N S}, \sigma_{0, N S}\right)$, (3) $\bar{l}_{N S} \equiv D_{H}\left(\sigma_{N S}, \bar{\sigma}_{0, N S}\right)$, (4) $\tilde{l}_{N S} \equiv l_{N S}+\bar{l}_{N S}$ $-l_{I}$. Therefore $\tilde{l}_{N S}$ is simply the number of positions where $\sigma_{N S}$ differs from both $\sigma_{0, N S}$ and $\bar{\sigma}_{0, N S}$.

Now, any $\sigma^{\prime}$ may be generated from any $\sigma$ by making the appropriate base changes. We can make changes to $\sigma$ as follows.

(1) Let $l_{1, S}$ denote the number of changes to $\sigma_{S}$ where $\sigma_{S}$ and $\sigma_{0, S}$ are identical. There are $\left(\begin{array}{c}L-l_{l}-l_{S} \\ l_{1, S}\end{array}\right)(2 S-1)^{l_{1, S}}$ possibilities for this set of changes.

(2) Let $l_{2, S}$ denote the number of changes to $\sigma_{S}$ back to the corresponding base in $\sigma_{0, S}$, where $\sigma_{S}$ and $\sigma_{0, S}$ are distinct. The degeneracy in this case is $\left(\begin{array}{c}l_{S} \\ l_{2, S}\end{array}\right)$.

(3) Let $l_{3, S}$ denote the number of changes to $\sigma_{S}$ to bases distinct from the corresponding bases in $\sigma_{0, S}$, where $\sigma_{S}$ and $\sigma_{0, S}$ are distinct. The degeneracy is $\left(\begin{array}{c}l_{S}-l_{2, S} \\ l_{3, S}\end{array}\right)(2 S-2)^{l_{3, S}}$.

(4) Let $l_{11, N S}$ denote the number of changes to $\sigma_{N S}$ where $\sigma_{N S}, \sigma_{0, N S}$ are identical, to bases other than the corresponding ones in $\bar{\sigma}_{0, N S}$. The degeneracy is $\left(\begin{array}{c}l_{l}-l_{N S} \\ l_{11, N S}\end{array}\right)(2 S-2)^{l_{11, N S}}$.

(5) Let $l_{12, N S}$ denote the number of changes to $\sigma_{N S}$ where $\sigma_{N S}, \sigma_{0, N S}$ are identical, to the corresponding bases in $\bar{\sigma}_{0, N S}$. The degeneracy is $\left(\begin{array}{c}l_{1}-l_{N S}-l_{11, N S} \\ l_{12, N S}\end{array}\right)$.

(6) Let $\bar{l}_{11, N S}$ denote the number of changes to $\sigma_{N S}$ where $\sigma_{N S}, \bar{\sigma}_{0, N S}$ are identical, to bases other than the corresponding

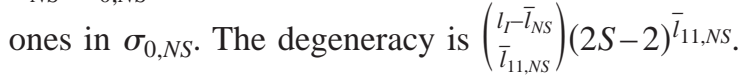

(7) Let $\bar{l}_{12, N S}$ denote the number of changes to $\sigma_{N S}$ where $\sigma_{N S}, \bar{\sigma}_{0, N S}$ are identical, to the corresponding bases in $\sigma_{0, N S}$. The degeneracy is $\left(\begin{array}{c}l_{I}-\bar{l}_{N S}-\bar{l}_{11, N S} \\ \bar{l}_{12, N S}\end{array}\right)$.

(8) Let $l_{2, N S}$ denote the number of changes to $\sigma_{N S}$, where $\sigma_{N S}$ is distinct from $\sigma_{0, N S}$ and $\bar{\sigma}_{0, N S}$, and the bases are changed to the corresponding bases in $\sigma_{N S, 0}$. The degeneracy is $\left(\begin{array}{c}\tilde{l}_{N S} \\ l_{2, N S}\end{array}\right)$.

(9) Let $\bar{l}_{2, N S}$ denote the number of changes to $\sigma_{N S}$, where $\sigma_{N S}$ is distinct from $\sigma_{0, N S}$ and $\bar{\sigma}_{0, N S}$, and the bases are changed to the corresponding bases in $\bar{\sigma}_{N S, 0}$. The degeneracy is $\left(\begin{array}{c}\tilde{l}_{N S}-l_{2, N S} \\ \tilde{l}_{2, N S}\end{array}\right)$.

(10) Let $l_{3, N S}$ denote the number of changes to $\sigma_{N S}$, where $\sigma_{N S}$ is distinct from $\sigma_{0, N S}$ and $\bar{\sigma}_{0, N S}$, and the bases are changed to bases other than the corresponding ones in $\sigma_{N S, 0}$ and $\bar{\sigma}_{N S, 0}$. The degeneracy is $\left(\begin{array}{c}\tilde{l}_{N S}-l_{2, N S}-\tilde{l}_{2, N S} \\ l_{3, N S}\end{array}\right)(2 S-3)^{l_{3, N S}}$.

The series of changes to $\sigma$ defined above yield a $\sigma^{\prime}$ which is at a Hamming distance of $l_{1, S}+l_{2, S}+l_{3, S}+l_{11, N S}+l_{12, N S}$ $+\bar{l}_{11, N S}+\bar{l}_{12, N S}+l_{2, N S}+\bar{l}_{2, N S}+l_{3, N S}$ from $\sigma$. Furthermore, the values of $l_{S}, l_{N S}, \bar{l}_{N S}$, and $\tilde{l}_{N S}$ for $\sigma^{\prime}$ are given by

$$
\begin{gathered}
l_{S}^{\prime}=l_{S}+l_{1, S}-l_{2, S}, \\
l_{N S}^{\prime}=l_{N S}+l_{11, N S}+l_{12, N S}-\bar{l}_{12, N S}-l_{2, N S}, \\
\bar{l}_{N S}^{\prime}=\bar{l}_{N S}+\bar{l}_{11, N S}+\bar{l}_{12, N S}-l_{12, N S}-\bar{l}_{2, N S}, \\
\tilde{l}_{N S}^{\prime}=\tilde{l}_{N S}+l_{11, N S}+\bar{l}_{11, N S}-l_{2, N S}-\bar{l}_{2, N S} .
\end{gathered}
$$

Now, we will assume that $y_{\sigma}$ depends only on $l_{S}, l_{N S}$, and $\bar{l}_{N S}$. At time $t=0$, we start the evolution by setting $y_{\sigma}=0$ for all $\sigma \neq \sigma_{0}, \bar{\sigma}_{0}$, and $y_{\sigma_{0}}=y_{\bar{\sigma}_{0}}=1 / 2$ if $\sigma_{0} \neq \bar{\sigma}_{0}$, and 1 if $\sigma_{0}=\bar{\sigma}_{0}$. Therefore, $y_{\sigma}=0$ unless $l_{S}=0$ and $l_{N S}=0$ or $\bar{l}_{N S}=0$. So certainly $y_{\sigma}$ depends only on $l_{S}, l_{N S}$, and $\bar{l}_{N S}$ at the start of the evolution. Also, by similar reasoning, we see that the fitness landscape $\left\{\kappa_{\sigma}\right\}$ also depends only on $l_{S}, l_{N S}, \bar{l}_{N S}$. If we can 
show that this implies that $d y_{\sigma} / d t$ depends only on $l_{S}, l_{N S}$, $\bar{l}_{N S}$, then $y_{\sigma}$ depends only on $l_{S}, l_{N S}, \bar{l}_{N S}$ throughout the evolution.

So, consider some time $t$ for which the $y_{\sigma}$ depend only on
$l_{S}, l_{N S}, \bar{l}_{N S}$, for all given $\sigma$ characterized by $l_{S}, l_{N S}, \bar{l}_{N S}$. Then

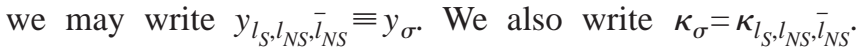
Summing over the contributions from the $\sigma^{\prime}$, obtained by the base changes described above, we obtain

$$
\begin{aligned}
& \frac{d y_{l_{S}, l_{N S}, \bar{l}_{N S}}}{d t}=2 \sum_{l_{1, S=0}}^{L-l_{I}-l_{S}} \sum_{l_{2, S=0}}^{l_{S}} \sum_{l_{3, S=0}}^{l_{S}-l_{2, S}} \sum_{l_{11, N S=0}}^{l_{I} l_{N S}} \sum_{l_{12, N S=0}}^{l_{l}-l_{N S}-l_{11, N S}} \sum_{\bar{l}_{11, N S=0}}^{l_{I}-\bar{l}_{N S}} \sum_{\bar{l}_{12, N S=0}}^{l_{I}-\bar{l}_{l S}-\bar{l}_{11, N S}} \sum_{l_{2, N S=0}}^{\tilde{l}_{N S}} \sum_{\bar{l}_{2, N S=0}}^{\tilde{l}_{N S}-l_{2, N S}} \tilde{l}_{l_{N S}-l_{2, N S}-\bar{l}_{2, N S}} \\
& \times\left(\begin{array}{c}
L-l_{I}-l_{S} \\
l_{1, S}
\end{array}\right)\left(\begin{array}{c}
l_{S} \\
l_{2, S}
\end{array}\right)\left(\begin{array}{c}
l_{S}-l_{2, S} \\
l_{3, S}
\end{array}\right)\left(\begin{array}{c}
l_{I}-l_{N S} \\
l_{11, N S}
\end{array}\right)\left(\begin{array}{c}
l_{I}-l_{N S}-l_{11, N S} \\
l_{12, N S}
\end{array}\right)\left(\begin{array}{c}
l_{I}-\bar{l}_{N S} \\
\bar{l}_{11, N S}
\end{array}\right) \\
& \times\left(\begin{array}{c}
l_{I}-\bar{l}_{N S}-\bar{l}_{11, N S} \\
\bar{l}_{12, N S}
\end{array}\right)\left(\begin{array}{c}
\tilde{l}_{N S} \\
l_{2, N S}
\end{array}\right)\left(\begin{array}{c}
\tilde{l}_{N S}-l_{2, N S} \\
\bar{l}_{2, N S}
\end{array}\right)\left(\begin{array}{c}
\tilde{l}_{N S}-l_{2, N S}-\bar{l}_{2, N S} \\
l_{3, N S}
\end{array}\right) \\
& \times(2 S-1)^{l_{1, S}(2 S-2)^{l_{3, S}}(2 S-2)^{l_{11, N S}}(2 S-2)^{\bar{l}_{11, N S}}(2 S-3)^{l_{3, N S}}} \\
& \times\left(\frac{\epsilon / 2}{2 S-1}\right)^{l_{1, S}+l_{2, S}+l_{3, S}+l_{11, N S}+\bar{l}_{11, N S}+l_{12, N S}+\bar{l}_{12, N S}+l_{2, N S}+\bar{l}_{2, N S}+l_{3, N S}} \\
& \times\left(1-\frac{\epsilon}{2}\right)^{L-l_{1, S}-l_{2, S}-l_{3, S}-l_{11, N S}-\bar{l}_{11, N S} l_{12, N S}-\bar{l}_{12, N S}-l_{2, N S}-\bar{l}_{2, N S} l_{3, N S}}
\end{aligned}
$$

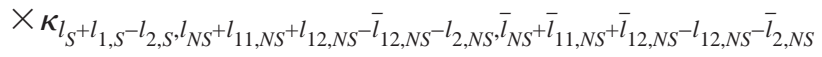

$$
\begin{aligned}
& \times y_{l_{S}+l_{1, S}} l_{2, S}, l_{N S}+l_{11, N S}+l_{12, N S}{ }^{-} \bar{l}_{12, N S}-l_{2, N S}, \bar{l}_{N S}+\bar{l}_{11, N S}+\bar{l}_{12, N S}{ }^{-l_{12, N S}}-\bar{l}_{2, N S}-\left[\kappa_{l_{S}, l_{N S}, \bar{l}_{N S}}+\bar{\kappa}(t)\right] y_{l_{S}, l_{N S}, \bar{l}_{N S}} .
\end{aligned}
$$

Note from the sum that $d y_{\sigma} / d t=d y_{\sigma^{\prime}} / d t$ for any two $\sigma, \sigma^{\prime}$ characterized by the same $l_{S}, l_{N S}$, and $\bar{l}_{N S}$. Therefore, the assumption that $y_{\sigma}$ is determined by $l_{S}, l_{N S}, \bar{l}_{N S}$ is justified.

We may sum over $l_{3, S}$ and $l_{3, N S}$ to obtain

$$
\begin{aligned}
& \frac{d y_{l_{S}, l_{N S}, \bar{l}_{N S}}}{d t}=2 \sum_{l_{1, S}=0}^{L-l_{l}-l_{S}} \sum_{l_{2, S}=0}^{l_{S}} \sum_{l_{11, N S}=0}^{l_{I}-l_{N S}} \sum_{l_{12, N S}=0}^{l_{l}-l_{N S}-l_{11, N S}} \sum_{\bar{l}_{11, N S}=0}^{l_{I} \bar{l}_{N S}} \sum_{\bar{l}_{12, N S}=0}^{l_{I}-\bar{l}_{N S}-\bar{l}_{11, N S}} \sum_{l_{2, N S}=0}^{\tilde{l}_{N S}} \sum_{\bar{l}_{2, N S}=0}^{\tilde{l}_{N S} l_{l_{2, N S}}} \\
& \times\left(\begin{array}{c}
L-l_{I}-l_{S} \\
l_{1, S}
\end{array}\right)\left(\begin{array}{c}
l_{S} \\
l_{2, S}
\end{array}\right)\left(\begin{array}{c}
l_{I}-l_{N S} \\
l_{11, N S}
\end{array}\right)\left(\begin{array}{c}
l_{I}-l_{N S}-l_{11, N S} \\
l_{12, N S}
\end{array}\right) \\
& \times\left(\begin{array}{c}
l_{I}-\bar{l}_{N S} \\
\bar{l}_{11, N S}
\end{array}\right)\left(\begin{array}{c}
l_{I}-\bar{l}_{N S}-\bar{l}_{11, N S} \\
\bar{l}_{12, N S}
\end{array}\right)\left(\begin{array}{c}
\tilde{l}_{N S} \\
l_{2, N S}
\end{array}\right)\left(\begin{array}{c}
\tilde{l}_{N S}-l_{2, N S} \\
\bar{l}_{2, N S}
\end{array}\right) \\
& \times(2 S-2)^{l_{11, N S}+\bar{l}_{11, N S}}\left(\frac{\boldsymbol{\epsilon}}{2}\right)^{l_{1, S}}\left(\frac{\epsilon / 2}{2 S-1}\right)^{l_{2, S}+l_{11, N S}+\bar{l}_{11, N S}+l_{12, N S}+\bar{l}_{12, N S}+l_{2, N S}+\bar{l}_{2, N S}} \\
& \times\left(1-\frac{\epsilon / 2}{2 S-1}\right)^{l_{S}-l_{2, S}}\left(1-\frac{\epsilon}{2 S-1}\right)^{\tilde{l}_{N S}-l_{2, N S}-\bar{l}_{2, N S}} \\
& \times\left(1-\frac{\epsilon}{2}\right)^{L-l_{S}-\tilde{l}_{N S}-l_{1, S}-l_{11, N S}-\bar{l}_{11, N S}-l_{12, N S}-\bar{l}_{12, N S}}
\end{aligned}
$$

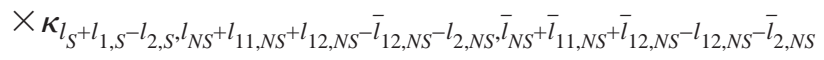

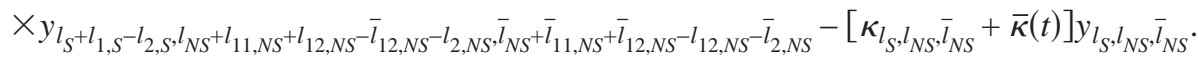


Now, the total number of sequences $\sigma$ characterized by the Hamming distances $l_{S}, l_{N S}$, and $\bar{l}_{N S}$ is given by $C_{l_{S}}, l_{N S}, \bar{l}_{N S}$

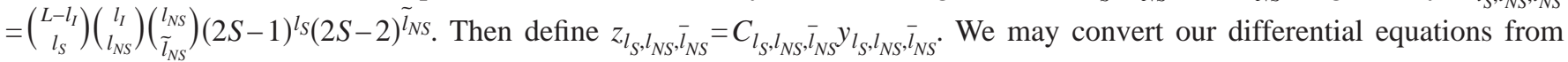
the $y$ to the $z$ representations. After some tedious algrebra, the final result is

$$
\begin{aligned}
& \frac{d z_{l_{S}, l_{N S}, \bar{l}_{N S}}}{d t}=2 \sum_{l_{1, S}=0}^{L-l_{l}-l_{S}} \sum_{l_{2, S}=0}^{l_{S}} \sum_{l_{11, N S}=0}^{l_{I}-l_{N S}} \sum_{l_{12, N S}=0}^{l_{I}-l_{N S}-l_{11, N S}} \sum_{\bar{l}_{11, N S}=0}^{l_{I}-\bar{l}_{N S}} \sum_{\bar{l}_{12, N S}=0}^{l_{I}-\bar{l}_{N S} \bar{l}_{11, N S}} \sum_{l_{2, N S}=0}^{\tilde{l}_{N S}} \sum_{\bar{l}_{2, N S}=0}^{\tilde{l}_{N S}-l_{2, N S}} \\
& \times\left(\begin{array}{c}
l_{1, S}+l_{S}-l_{2, S} \\
l_{1, S}
\end{array}\right)\left(\frac{\epsilon / 2}{2 S-1}\right)^{l_{1, S}}\left(1-\frac{\epsilon / 2}{2 S-1}\right)^{l_{S}-l_{2, S}} \\
& \times\left(\begin{array}{c}
L-l_{I}-l_{S}-l_{1, S}+l_{2, S} \\
l_{2, S}
\end{array}\right)\left(\frac{\epsilon}{2}\right)^{l_{2, S}} \\
& \times\left(\begin{array}{c}
\tilde{l}_{N S}-l_{2, N S}-\bar{l}_{2, N S}+l_{11, N S}+\bar{l}_{11, N S} \\
\bar{l}_{11, N S}
\end{array}\right)\left(\begin{array}{c}
\tilde{l}_{N S}-l_{2, N S}-\bar{l}_{2, N S}+l_{11, N S} \\
l_{11, N S}
\end{array}\right) \\
& \times\left(\frac{\epsilon / 2}{2 S-1}\right)^{l_{11, N S}+\bar{l}_{11, N S}}\left(1-\frac{\epsilon}{2 S-1}\right)^{\tilde{l}_{N S}-l_{2, N S}-\bar{l}_{2, N S}} \\
& \times\left(\begin{array}{c}
l_{I}-l_{N S}-l_{11, N S}-l_{12, N S}+l_{2, N S}+\bar{l}_{12, N S} \\
\bar{l}_{12, N S}
\end{array}\right)\left(\begin{array}{c}
l_{I}-l_{N S}-l_{11, N S}-l_{12, N S}+l_{2, N S} \\
l_{2, N S}
\end{array}\right) \\
& \times\left(\frac{\epsilon / 2}{2 S-1}\right)^{\bar{l}_{12, N S}+l_{2, N S}} \\
& \times\left(\begin{array}{c}
l_{I}-\bar{l}_{N S}-\bar{l}_{11, N S}-\bar{l}_{12, N S}+l_{12, N S}+\bar{l}_{2, N S} \\
\bar{l}_{2, N S}
\end{array}\right)\left(\begin{array}{c}
l_{I}-\bar{l}_{N S}-\bar{l}_{11, N S}-\bar{l}_{12, N S}+l_{12, N S} \\
l_{12, N S}
\end{array}\right) \\
& \times\left(\frac{\epsilon / 2}{2 S-1}\right)^{\bar{l}_{2, N S}+l_{12, N S}}(2 S-2)^{l_{2, N S} \bar{l}_{2, N S}}\left(1-\frac{\boldsymbol{\epsilon}}{2}\right)^{L-l_{S}-\tilde{l}_{N S}-l_{1, S}-l_{11, N S}-\bar{l}_{11, N S}-l_{12, N S}-\bar{l}_{12, N S}}
\end{aligned}
$$

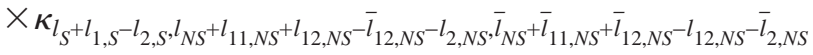

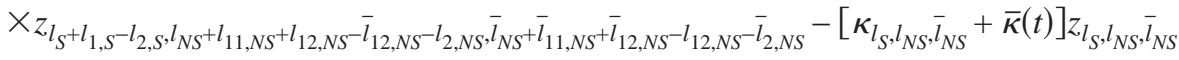

\section{The infinite sequence length equations}

We are now in a position to derive the infinite sequence length form of the quasispecies equations. We allow $L \rightarrow \infty$ while keeping $\mu \equiv L \epsilon$ fixed. Furthermore, let us define $f_{I}$ $=l_{I} / L$, so $f_{I}$ is the fraction of bases in $\sigma_{0}$ and $\bar{\sigma}_{0}$ which differ. If we let $p\left(f_{I}\right)$ denote the probability density for $f_{I}$, then in the limit of infinite sequence length we obtain that $p\left(f_{I}\right)$ $\rightarrow \delta\left\{f_{I}-[1-(1 / 2 S)]\right\}$, where $\delta$ is the Dirac $\delta$ function. Therefore, we take $f_{I}=1-1 / 2 S$ in the $L \rightarrow \infty$ limit.

A slight complication arises in the infinite sequence limit, namely, that $l_{I}=f_{I} L \rightarrow \infty$ as $L \rightarrow \infty$. This means that it is impossible for $l_{N S}$ and $\bar{l}_{N S}$ to simultaneously be finite. For if $l_{N S}$ is finite, then $\bar{l}_{N S}=l_{I}-l_{N S}+\widetilde{l}_{N S}=\infty$ and vice versa. The appropriate way to solve these equations is therefore to solve for

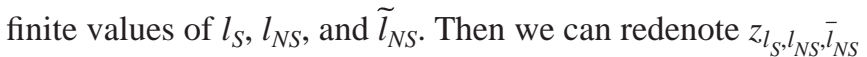

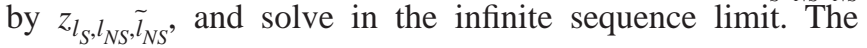
symmetry of the landscape allows us to obtain the finite $\bar{l}_{N S}$ population fractions as well, since the population fraction for finite $l_{S}, \bar{l}_{N S}$, and $\tilde{l}_{N S}$ is then simply given by $z_{l_{S}}, \bar{l}_{N S}, \tilde{l}_{N S}$.

In the following subsection, we show that as $L \rightarrow \infty$, the only terms which survive the limiting process are the $l_{1, S}$ $=l_{11, N S}=\bar{l}_{11, N S}=\bar{l}_{2, N S}=l_{12, N S}=0$ terms. We also have

$$
\begin{gathered}
\left(\begin{array}{c}
L-l_{I}-l_{S}+l_{2, S} \\
l_{2, S}
\end{array}\right)\left(\frac{\epsilon}{2}\right)^{l_{2, S}} \rightarrow \frac{1}{l_{2, S} !}\left(\frac{\left(1-f_{I}\right) \mu}{2}\right)^{l_{2, S}}, \text { (A5) } \\
\left(\begin{array}{c}
l_{I}-l_{N S}+l_{2, N S}+\bar{l}_{12, N S} \\
\bar{l}_{12, N S}
\end{array}\right)\left(\begin{array}{c}
l_{I}-l_{N S}+l_{2, N S} \\
l_{2, N S}
\end{array}\right)\left(\frac{\epsilon / 2}{2 S-1}\right)^{\bar{l}_{12, N S}+l_{2, N S}} \\
\rightarrow \frac{1}{l_{2, N S} ! \bar{l}_{12, N S} !}\left(\frac{f_{I} \mu}{2(2 S-1)}\right)^{\bar{l}_{12, N S}+l_{2, N S}},
\end{gathered}
$$




$$
\left(1-\frac{\boldsymbol{\epsilon}}{2}\right)^{L-l_{S}-\tilde{l}_{N S}-l_{1, S}-l_{11, N S}-\bar{l}_{11, N S}-l_{12, N S}-\bar{l}_{12, N S}} \rightarrow e^{-\mu / 2} .
$$

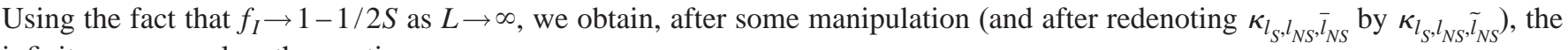
infinite sequence length equations

$$
\begin{aligned}
& \frac{d z_{l_{S}, l_{N S}, \tilde{l}_{N S}}}{d t}=2 e^{-\mu / 2} \sum_{l_{1, S=0}}^{l_{S}} \sum_{l_{1, N S=0}}^{l_{N S}-\tilde{l}_{N S}} \sum_{l_{2, N S=0}}^{\tilde{l}_{N S}} \frac{1}{l_{1, S} ! l_{1, N S} ! l_{2, N S} !}\left(\frac{\mu}{4 S}\right)^{l_{1, S}+l_{1, N S}+l_{2, N S}}(2 S-2)^{l_{2, N S}}
\end{aligned}
$$

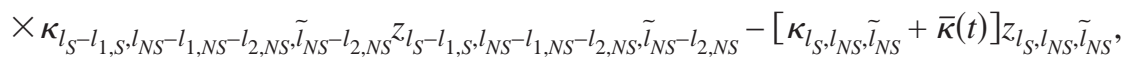

where we have redenoted $l_{2, S}$ by $l_{1, S}$, and $\bar{l}_{12, N S}$ by $l_{1, N S}$.

It should be clear that $z_{0,0,0}=y_{\sigma_{0}}$. Therefore, $2 z_{0,0,0}$ is the total fraction of the population with genome $\left\{\sigma_{0}, \bar{\sigma}_{0}\right\}$. This gives, $\bar{\kappa}(t)=2(k-1) z_{0,0,0}+1$.

Now, as $L \rightarrow \infty$, the sequences $\sigma_{0}$ and $\bar{\sigma}_{0}$ become infinitely separated. Therefore, we expect that the values of $z_{l_{S}, l_{N S}, \tilde{l}_{N S}}$ for finite $l_{S}, l_{N S}, \tilde{l}_{N S}$ to be dictated by the single fitness peak at $\sigma_{0}$. Thus, for large $L$, we expect to obtain a locally single fitness peak model in which we can then assume that $y_{\sigma}$ depends only on the Hamming distance $l_{S}+l_{N S}$ to $\sigma_{0}$. In the following subsection, we prove this rigorously. We may then group the population into Hamming classes, as with the single fitness peak for conservative replication. Specifically,

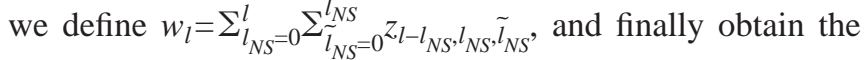
infinite sequence length equations given by Eq. (17).

\section{Additional calculational details}

\section{a. Derivation of the infinite sequence length equations from the finite sequence length equatiions}

In this appendix, we derive the infinite sequence length

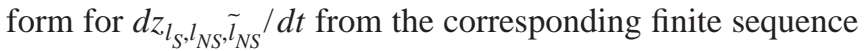
length equations. Before proceeding, however, we derive some basic inequalities which we will need to use. First of all, note that each $z_{l_{S}, l_{N S}, \tilde{l}_{N S}}$ must be $\leqslant 1$. Furthermore, note

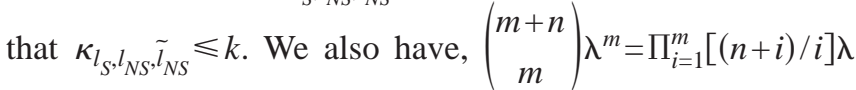
$\leqslant[(n+1) \lambda]^{m}$, and $(1-\lambda)^{n} \leqslant 1$ for $\lambda \in[0,1]$.

We wish to show that in the limit of $L \rightarrow \infty$, the only terms which contribute to the dynamical equations are the $l_{1, S}$ $=l_{11, N S}=\bar{l}_{11, N S}=\bar{l}_{2, N S}=l_{12, N S}=0$ terms. We prove this by showing that for each of the above indices, the total contribution from all the nonzero terms becomes arbitrarily small as $L$ $\rightarrow \infty$ with $\mu=L \epsilon$ held fixed.

So, we start with the $l_{1, S}$ index. From the inequalities given above, we may note that the summand of Eq. (A4),

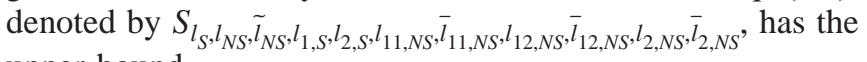
upper bound

$$
\begin{aligned}
& S_{l_{S}, l_{N S}, \tilde{l}_{N S}, l_{1, S}, l_{2, S}, l_{11, N S}, \bar{l}_{11, N S}, l_{12, N S}, \bar{l}_{12, N S}, l_{2, N S}, \bar{l}_{2, N S}} \\
& \quad \leqslant k\left[\left(l_{S}+1\right)\left(\frac{\epsilon / 2}{2 S-1}\right)\right]^{l_{1, S}}\left((L+1) \frac{\epsilon}{2}\right)^{l_{2, S}}\left[\left(\tilde{l}_{N S}+1\right)\right. \\
& \left.\quad \times\left(\frac{\epsilon / 2}{2 S-1}\right)\right]^{l_{11, N S}}\left[\left(\tilde{l}_{N S}+1\right)\left(\frac{\epsilon / 2}{2 S-1}\right)^{\bar{l}_{11, N S}}\right] \\
& -\left[\left(l_{I}+1\right)\left(\frac{\epsilon / 2}{2 S-1}\right)\right]^{\bar{l}_{12, N S}}\left[\left(l_{I}+1\right)\left(\frac{2 S-2}{2 S-1} \frac{\epsilon}{2}\right)\right]^{l_{2, N S}}\left[\left(l_{N S}\right.\right. \\
& \left.\left.\quad-\tilde{l}_{N S}+1\right)\left(\frac{2 S-2}{2 S-1} \frac{\epsilon}{2}\right)^{\bar{l}_{2, N S}}\right]\left[\left(l_{N S}-\tilde{l}_{N S}+1\right)\left(\frac{\epsilon / 2}{2 S-1}\right)^{l_{12, N S}}\right] .
\end{aligned}
$$

Now, at fixed $\mu$, choose $L$ to be sufficiently large so that $(L+1)(\epsilon / 2)=1 / 2(\mu+\epsilon)<\mu$. Then certainly $\left(l_{I}+1\right)$ $\times[\epsilon / 2 /(2 S-1)]<\mu /(2 S-1)$. We then have

$$
\begin{aligned}
& { }_{L-l_{I}-l_{S}} \quad l_{S} \quad l_{I}-l_{N S} \quad l_{I}-l_{N S}-l_{11, N S} l_{N S}-\tilde{l}_{N S} l_{N S}-\tilde{l}_{N S}-\bar{l}_{11, N S} \quad \tilde{l}_{N S} \quad \tilde{l}_{N S}-l_{2, N S} \\
& \sum_{l_{1, S}=1} \sum_{l_{2, S}=0} \sum_{l_{11, N S}=0} \sum_{l_{12, N S}=0} \sum_{\bar{l}_{11, N S}=0} \sum_{\bar{l}_{12, N S}=0} \sum_{l_{2, N S}=0} \sum_{\bar{l}_{2, N S}=0}^{2, N S}
\end{aligned}
$$

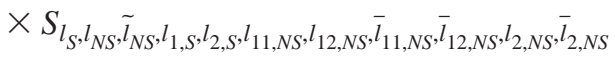

$$
\begin{aligned}
& \leqslant k \sum_{l_{1, S}=1}^{L-l_{I}-l_{S}}\left[\left(l_{S}+1\right)\left(\frac{\epsilon / 2}{2 S-1}\right)\right]{ }^{l_{1, S}} \sum_{l_{2, S}=0}^{l_{S}} \mu^{l_{2, S}} \sum_{l_{11, N S}=0}^{l_{I}-l_{N S}}\left[\left(\tilde{l}_{N S}+1\right)\right. \\
& \left.\times\left(\frac{\epsilon / 2}{2 S-1}\right)\right]^{l_{11, N S}} \times \sum_{l_{12, N S}=0}^{l_{I}-l_{N S}}\left[\left(l_{N S}-\tilde{l}_{N S}+1\right)\right. \\
& \left.\times\left(\frac{\epsilon / 2}{2 S-1}\right)\right] \sum_{\bar{l}_{11, N S}=0}^{l_{12, N S}}\left[\left(\tilde{l}_{N S}+1\right)\left(\frac{\epsilon / 2}{2 S-1}\right)\right]^{l_{N S}-\tilde{l}_{N S}}[ \\
& \times \sum_{\bar{l}_{12, N S}=0}^{l_{N S} \tilde{l}_{N S}}\left(\frac{\mu}{2 S-1}\right)^{\bar{l}_{12, N S}} \sum_{l_{2, N S}=0}^{\tilde{l}_{N S}} \mu^{l_{2, N S}} \sum_{\bar{l}_{2, N S}=0}^{\tilde{l}_{N S}}\left[\left(l_{N S}-\tilde{l}_{N S}+1\right)\right. \\
& \left.\times\left(\frac{\boldsymbol{\epsilon}}{2}\right)\right]^{\bar{l}_{2, N S}} \text {. }
\end{aligned}
$$

Now, let $A_{l} \equiv \sum_{k=0}^{l} \mu^{k}$. Also, note that $\sum_{n=m}^{\infty} \lambda^{n}=\lambda^{m} /(1-\lambda)$, for $|\lambda|<1$. Therefore, note that an upper bound for the product given above is simply, $k A_{l_{S}+l_{N S}}^{3}\left[\left(l_{S}+l_{N S}+1\right)(\epsilon / 2)\right] /\left[1-\left(l_{S}\right.\right.$ 
$\left.\left.+l_{N S}+1\right)(\epsilon / 2)\right]^{5}$. Therefore, as $L \rightarrow \infty$, so that $\epsilon \rightarrow 0$ in such a way that $\mu$ is fixed, we see that the contribution of the $l_{1, S}>0$ terms to the evolution dynamics approaches 0 . Therefore, we need only consider the $l_{1, S}=0$ terms in the limit of infinite sequence length. Using a similar argument to the one given above, we can systematically eliminate the contributions from the $l_{11, N S}, l_{12, N S}, \bar{l}_{11, N S}, \bar{l}_{2, N S}>0$ as well. This establishes the infinite sequence length form of our differential equations. We should note that convergence to the infinite sequence length form is not uniform, as can be seen by the $l_{S}+l_{N S}$ dependence of our upper bound.

\section{b. Simplification of the infinite sequence length equations}

We wish to show that, as $L \rightarrow \infty$, we may assume that $y_{l_{S}, l_{N S}, \tilde{l}_{N S}}$ becomes dependent only on $l_{S}+l_{N S}$, which will thereby allow us to considerably simplify the infinite sequence length equations [Eq. (A4)]. To proceed with this simplification, let us first determine the effect that $y_{l_{S}, l_{N S}, \tilde{l}_{N S}}$

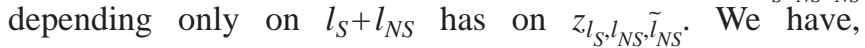

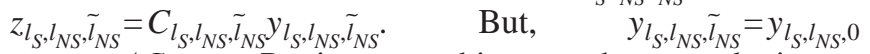
$=z_{l_{S}, l_{N S}, 0} / C_{l_{S}, l_{N S}, 0}$. Putting everything together, we obtain

$$
z_{l_{S}, l_{N S}, \tilde{l}_{N S}}=\left(\begin{array}{c}
l_{N S} \\
\tilde{l}_{N S}
\end{array}\right)(2 S-2)^{\tilde{l}_{N S}} z_{l_{S}, l_{N S}, 0} .
$$

A similar procedure yields

$$
\begin{aligned}
z_{l_{S}, l_{N S}, 0}= & \left(\begin{array}{c}
l_{S}+l_{N S} \\
l_{N S}
\end{array}\right) \frac{l_{I} !\left(L-l_{I}-l_{S}-l_{N S}\right) !}{\left(l_{I}-l_{N S}\right) !\left(L-l_{I}-l_{S}\right) !} \\
& \times(2 S-1)^{-l_{N S}} z_{l_{S}+l_{N S}, 0,0} .
\end{aligned}
$$

As $L, l_{I} \rightarrow \infty$, we get

$$
\begin{aligned}
\frac{l_{I} !}{\left(l_{I}-l_{N S}\right) !} \frac{\left(L-l_{I}-l_{S}-l_{N S}\right) !}{\left(L-l_{I}-l_{S}\right) !} & \rightarrow\left(\frac{l_{I}}{L-l_{I}}\right)^{l_{N S}}=\left(\frac{f_{I}}{1-f_{I}}\right)^{l_{N S}} \\
& =(2 S-1)^{l_{N S}} .
\end{aligned}
$$

giving, $z_{l_{S}, l_{N S}, 0}=\left(\begin{array}{c}l_{S}+l_{N S} \\ l_{N S}\end{array}\right) z_{l_{S}+l_{N S}, 0,0}$. Therefore,

$$
z_{l_{S}, l_{N S}, \tilde{l}_{N S}}=\left(\begin{array}{c}
l_{S}+l_{N S} \\
l_{N S}
\end{array}\right)\left(\begin{array}{c}
l_{N S} \\
\tilde{l}_{N S}
\end{array}\right)(2 S-2)^{\tilde{l}_{N S}} z_{l_{S}+l_{N S}, 0,0} .
$$

We wish to show that it is this relation which is preserved by the evolution equations. Note that at time $t=0$, we have $z_{l_{S}, l_{N S}, \tilde{l}_{N S}}=1 / 2 \delta_{l_{S}+l_{N S}, 0}$, so that this relation holds at $t=0$. If we can show that if this relation holds for all $z_{l_{S}, l_{N S}, \tilde{l}_{N S}}$ at some time $t$, then it holds for $d z_{l_{l}, l_{N S}, \tilde{l}_{N S}} / d t$, it follows that it holds throughout the evolution.
We note also that $\kappa_{l_{S}, l_{N S}, \tilde{l}_{N S}}$ only depends on $l_{S}+l_{N S}$ in the limit of infinite sequence length. Therefore, we may define

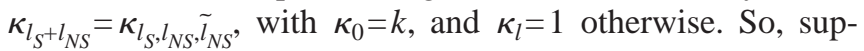
pose at some time $t$ we have that Eq. (A14) holds for all $l_{S}, l_{N S}, \tilde{l}_{N S}$. Then, after switching notation from $l_{S}, l_{N S}, \tilde{l}_{N S}$ to $p, q, r$, we have

$$
\begin{aligned}
\frac{d z_{p, q, r}}{d t}= & \sum_{j=0}^{p} \sum_{k=0}^{q-r} \sum_{l=0}^{r} \frac{1}{j ! k ! l !}\left(\frac{\mu}{4 S}\right)^{j+k+l}(2 S-2)^{l} \\
& \times \kappa_{p+q-j-k-l}\left(\begin{array}{c}
p+q-j-k-l \\
p-j
\end{array}\right)\left(\begin{array}{c}
q-k-l \\
r-l
\end{array}\right) \\
& \times(2 S-2)^{r-l} z_{p+q-j-k-l, 0,0}-\left[\kappa_{p, q, r}+\bar{\kappa}(t)\right] \\
& \times\left(\begin{array}{c}
p+q \\
p
\end{array}\right)\left(\begin{array}{l}
q \\
r
\end{array}\right)(2 S-2)^{r} z_{p+q, 0,0} \\
= & \left(\begin{array}{c}
p+q \\
p
\end{array}\right)\left(\begin{array}{l}
q \\
r
\end{array}\right)(2 S-2)^{r} \\
& \times \sum_{m=0}^{p+q} \frac{1}{m !}\left(\frac{\mu}{4 S}\right)^{m} \kappa_{p+q-m} z_{p+q-m, 0,0} \\
& \times\left[\begin{array}{c}
1 \\
\left(\begin{array}{c}
p+q \\
m
\end{array}\right)
\end{array} \sum_{j+k+l=m,(j, k, l) \in[0, p] \times[0, q-r] \times[0, r]}\left(\begin{array}{c}
p \\
j
\end{array}\right)\right. \\
& \left.\times\left(\begin{array}{c}
q-r \\
k
\end{array}\right)\left(\begin{array}{l}
r \\
l
\end{array}\right)\right]-\left[\begin{array}{c}
\left.\kappa_{p, q, r}+\bar{\kappa}(t)\right] \\
p+q \\
p
\end{array}\right)\left(\begin{array}{c}
q \\
r
\end{array}\right) \\
& \times(2 S-2)^{r} z_{p+q, 0,0} \\
= & \left(\begin{array}{c}
p+q \\
p
\end{array}\right)\left(\begin{array}{l}
q \\
r
\end{array}\right)(2 S-2)^{r} \frac{d z_{p+q, 0,0}}{d t} .
\end{aligned}
$$

The last two lines are derived by noting that the product

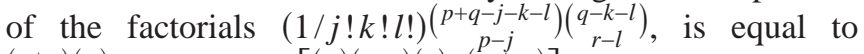
$\left(\begin{array}{c}p+q \\ p\end{array}\right)\left(\begin{array}{c}q \\ r\end{array}\right)[1 /(j+k+l) !]\left[\left(\begin{array}{c}( \\ j\end{array}\right)\left(\begin{array}{c}q-r \\ k\end{array}\right)\left(\begin{array}{c}r \\ l\end{array}\right) /\left(\begin{array}{c}p-j \\ j+k+l\end{array}\right)\right]$, and then by noting that $\left.\quad \sum_{j+k+l=m,(j, k, l) \in[0, p] \times[0, q-r] \times[0, r]} \begin{array}{c}p \\ j\end{array}\right)\left(\begin{array}{c}q-r \\ k\end{array}\right)\left(\begin{array}{c}r \\ l\end{array}\right)=\left(\begin{array}{c}p+q \\ m\end{array}\right)$. This relation can be derived by expanding $(x+1)^{p+q}$ in two different ways: First by direct expansion using the binomial theorem, and second by expanding $(x+1)^{p},(x+1)^{q-r},(x+1)^{r}$ separately, and then taking the product. Matching powers of $x$ yields the relation given above.

Note that that we have shown that $z_{p, q, r}=\left(\begin{array}{c}p+q \\ p\end{array}\right)\left(\begin{array}{c}q \\ r\end{array}\right)(2 S$ $-2)^{r} z_{p+q, 0,0}$ for all $p, q, r$ throughout the evolution. Then given some $l$, let us collect all the population at Hamming distance $l$ from $\sigma_{0}$ by defining $w_{l}=\sum_{m=0}^{l} \sum_{r=0}^{m} z_{l-m, m, r}$. We then have, $w_{l}=z_{l, 0,0} \sum_{m=0}^{l} \sum_{r=0}^{m}\left(\begin{array}{l}l \\ m\end{array}\right)\left(\begin{array}{c}m \\ r\end{array}\right)(2 S-2)^{r}=(2 S)^{l} z_{l, 0,0}$. Therefore, using the expression for $d z_{l, 0,0} / d t$, we immediately obtain the infinite sequence length equations given by Eq. (17). 
[1] M. Eigen, Naturwissenschaften 58, 465 (1971).

[2] M. Eigen, J. McCaskill, and P. Schuster, Adv. Chem. Phys. 75, 149 (1989).

[3] J. Swetina and P. Schuster, Biophys. Chem. 16, 329 (1982).

[4] P. Tarazona, Phys. Rev. A 45, 6038 (1992).

[5] S. Galluccio, Phys. Rev. E 56, 4526 (1997).

[6] R. Pastor-Satorras and R. Sole, Phys. Rev. E 64, 051909 (2001).

[7] S. Altmeyer and J. McCaskill, Phys. Rev. Lett. 86, 5819 (2001).

[8] S. Franz and L. Peliti, J. Phys. A 30, 4481 (1997).

[9] P. R. A. Campos and J. F. Fontanari, Phys. Rev. E 58, 2664
(1998).

[10] D. Alves and J. F. Fontanari, Phys. Rev. E 57, 7008 (1998). [11] M. Nilsson and N. Snoad, Phys. Rev. E 65, 031901 (2002).

[12] M. Nilsson and N. Snoad, Phys. Rev. Lett. 84, 191 (2000).

[13] E. Tannenbaum, E. J. Deeds, and E. I. Shakhnovich, Phys. Rev. Lett. 91, 138105 (2003).

[14] E. Tannenbaum and E. I. Shakhnovich, Phys. Rev. E 69, 011902 (2004).

[15] M. Kimura and T. Maruyama, Genetics 54, 1337 (1966).

[16] D. Voet and J. Voet, Biochemistry, 2nd ed. (J. Wiley, New York, 1995). 